\title{
Saturn's Northern Hemisphere Ribbon: Simulations and Comparison with the Meandering Gulf Stream
}

\author{
KUNIO M. SAYANAGI \\ Division of Geological and Planetary Sciences, California Institute of Technology, Pasadena, California \\ RAÚl MORALES-JUBERÍAS \\ Department of Physics, New Mexico Institute of Mining and Technology, Socorro, New Mexico \\ ANDREW P. INGERSOLL \\ Division of Geological and Planetary Sciences, California Institute of Technology, Pasadena, California
}

(Manuscript received 11 September 2009, in final form 18 March 2010)

\begin{abstract}
Voyager observations of Saturn in 1980-81 discovered a wavy feature engirdling the planet at $47^{\circ} \mathrm{N}$ planetographic latitude. Its latitude coincides with that of an eastward jet stream, which is the second fastest on Saturn after the equatorial jet. The $47^{\circ} \mathrm{N}$ jet's wavy morphology is unique among the known atmospheric jets on the gas giant planets. Since the Voyagers, it has been seen in every high-resolution image of this latitude for over 25 years and has been termed the Ribbon. The Ribbon has been interpreted as a dynamic instability in the jet stream. This study tests this interpretation and uses forward modeling to explore the observed zonal wind profile's stability properties. Unforced, initial-value numerical experiments are performed to examine the nonlinear evolution of the jet stream. Parameter variations show that an instability occurs when the $47^{\circ} \mathrm{N}$ jet causes reversals in the potential vorticity (PV) gradient, which constitutes a violation of the Charney-Stern stability criterion. After the initial instability development, the simulations demonstrate that the instability's amplitude nonlinearly saturates to a constant when the eddy generation by the instability is balanced by the destruction of the eddies. When the instability saturates, the zonal wind profile approaches neutral stability according to Arnol'd's second criterion, and the jet's path meanders in a Ribbon-like manner. It is demonstrated that the meandering of the $47^{\circ} \mathrm{N}$ jet occurs over a range of tropospheric static stability and background wind speed. The results here show that a nonlinearly saturated shear instability in the $47^{\circ} \mathrm{N}$ jet is a viable mechanism to produce the Ribbon morphology. Observations do not yet have the temporal coverage to confirm the creation and destruction of eddies, but these simulations predict that this is actively occurring in the Ribbon region. Similarities exist between the behaviors found in this model and the dynamics of PV fronts studied in the context of meandering western boundary currents in Earth's oceans. In addition, the simulations capture the nonlinear aspects of a new feature discovered by the Cassini Visual and Infrared Mapping Spectrometer (VIMS), the String of Pearls, which resides in the equatorward tip of the $47^{\circ} \mathrm{N}$ jet. The Explicit Planetary Isentropic Coordinate (EPIC) model is used herein.
\end{abstract}

\section{Introduction}

The Voyager observations of Saturn revealed a wavy dark streak meandering in the light-colored band in the visible wavelengths around the $47^{\circ} \mathrm{N}$ planetographic latitude that completely encircled the planet (Smith et al.

Corresponding author address: Kunio M. Sayanagi, MC 150-21, Division of Geological and Planetary Sciences, California Institute of Technology, Pasadena, CA 91125.

E-mail: sayanagi@gps.caltech.edu
1982). This feature has since been termed the Ribbon, and its location coincides with the peak of an eastward zonal jet. The feature is sandwiched between cloud patterns that exhibit streaks suggesting their motions to be cyclonic to the north and anticyclonic to the south (Fig. 1); the cyclones and anticyclones alternate their positions in longitude, and the Ribbon's path conforms around the patches of cyclonic and anticyclonic circulations (it is unclear whether they are vortices with closed streamlines). The path of the Ribbon jet buckles in some places, as shown in Fig. 2, although it is unclear whether 


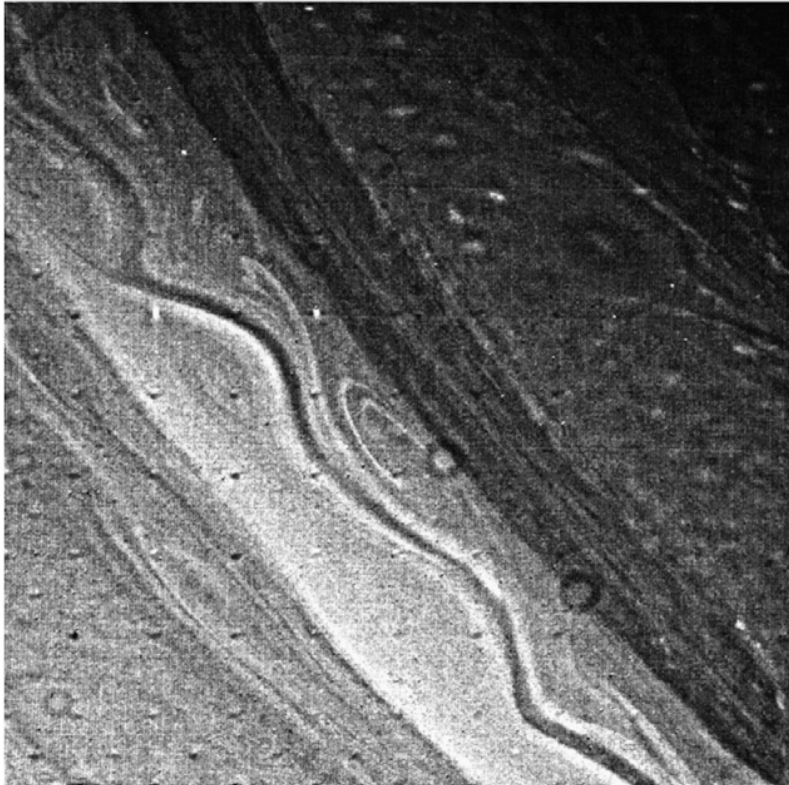

FIG. 1. Voyager 2 image of the Ribbon region, captured on 23 Aug 1981 (PIA01378). The Ribbon is the dark sinuous streak in the light band running diagonally in the image from the upper left to the lower right corners. North is to the upper right.

such features are long lived. Fourier analyses of the spatial oscillations indicate that it is not dominated by any single zonal wavelength, which matches its visual impression (Sromovsky et al. 1983; Godfrey and Moore 1986). The meandering propagates in the direction of the zonal wind. Sromovsky et al. (1983)'s measurements hint that the meandering's crests and troughs may not propagate coherently for longer than several Saturnian rotations, and thus its spectrum may be temporally variable. Sánchez-Lavega (2002) estimated the top of the haze layer at the Ribbon latitude to be $\sim 100 \mathrm{hPa}$, which he interpreted as the top of the Ribbon feature. Godfrey and Moore (1986) estimated that the Ribbon extends at least down to the altitude of the 170-hPa level.

The zonal mean speed of the $47^{\circ} \mathrm{N}$ jet is about $150 \mathrm{~m} \mathrm{~s}^{-1}$ eastward during the Voyager flybys, measured by Sánchez-Lavega et al. (2000) using the cloud-tracking method. This zonal-mean zonal wind speed is approximately equal to the phase speed of the Ribbon measured by Sromovsky et al. (1983); if true, this means that the Ribbon is stationary with respect to the local wind. However, Sánchez-Lavega et al. (2000)'s data points have a substantial scatter at around $47^{\circ} \mathrm{N}$ latitude, ranging between $\sim 200$ and $\sim 100 \mathrm{~m} \mathrm{~s}^{-1}$, suggesting that this is a region of substantial eddy activity. Sayanagi and Showman (2007) showed that atmospheric waves can alter the apparent motions of clouds and thus the outcome of cloudtracking measurement. Although it is unclear whether

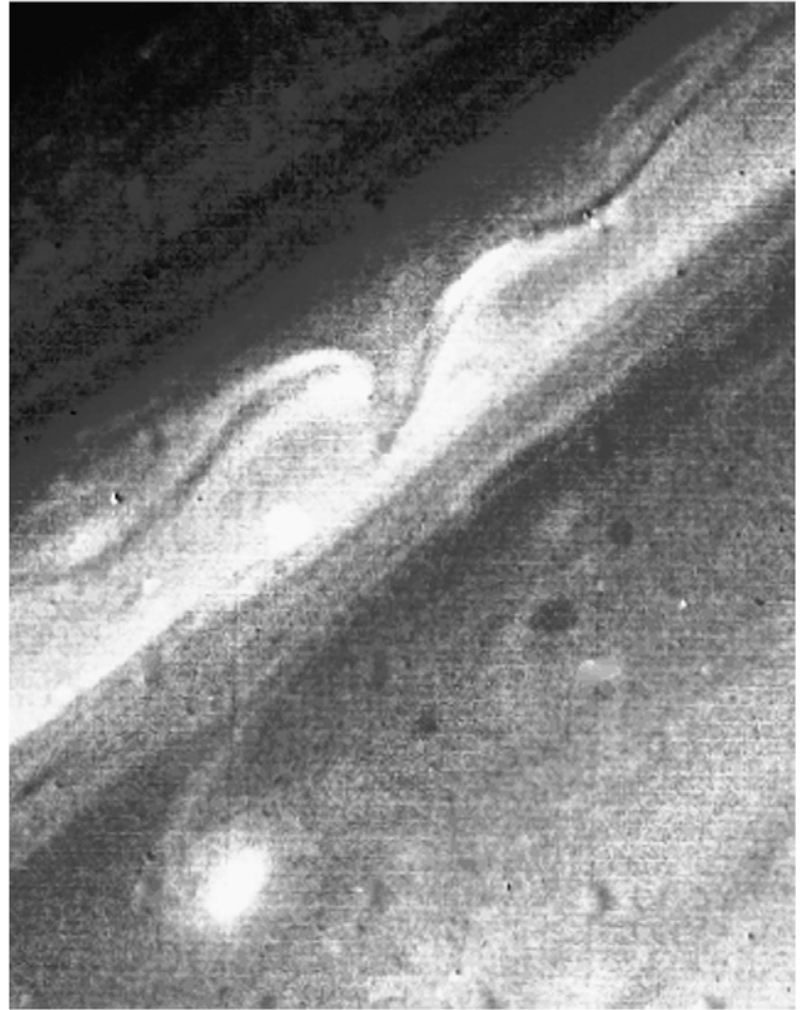

FIG. 2. Voyager 2 image of the Ribbon region, captured on 20 Aug 1981 (PIA01961), showing a buckling in the Ribbon. North is to the upper left.

the measurements by Sánchez-Lavega et al. (2000) were sensitive to wave motions, the match between the jet speed and the zonal phase speed of the Ribbon raises this possibility.

Through linear analysis, Godfrey and Moore (1986) showed that the thermal structure observed by the Voyagers makes the $47^{\circ} \mathrm{N}$ zonal jet susceptible to baroclinic instability. Their investigation showed that baroclinic instabilities can excite the wave modes observed in the Ribbon, and they reasoned that the Ribbon is a manifestation of the baroclinic instability. If the Ribbon is indeed a result of a form of dynamic instability, it is remarkable because the amplitude of the instability seems to be approximately constant and does not seem to grow; consequently, the instability does not destroy the jet. The results of a linear stability analysis such as that by Godfrey and Moore (1986) are applicable only to the initial infinitesimal growth of an instability mode. Despite the hypothesis that the Ribbon arises from dynamic instability, it is a long-lived feature; it is found in Hubble Space Telescope images captured in 1994-95 (Sánchez-Lavega 2002) and it can also be seen in recent Cassini images of Saturn. If the Ribbon is indeed caused by shear instabilities, the feature's longevity requires an 


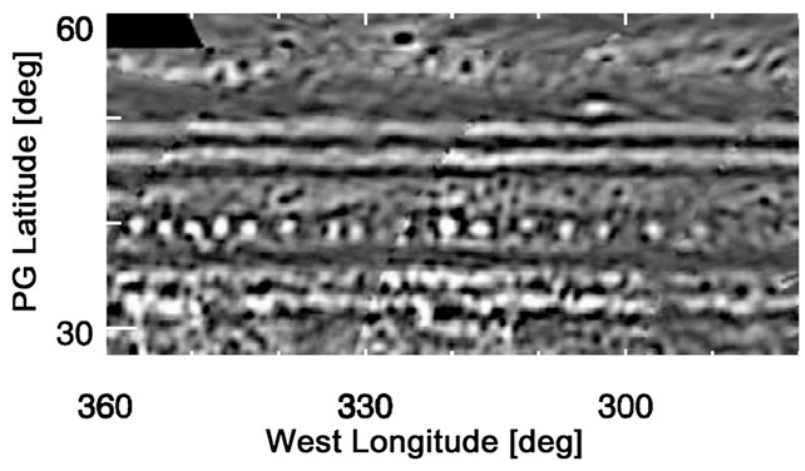

FIG. 3. Cassini VIMS 5- $\mu \mathrm{m}$ image of the Ribbon region and the SoPs, from Choi et al. (2009). The Ribbon region, centered near $47^{\circ} \mathrm{N}$, appears as the dark streak sandwiched between two bright bands in this image. The SoPs is the $\sim 15$ bright dots that line up at $40^{\circ} \mathrm{N}$.

explanation for what halts the growth of the instability and maintains the jet in its observed meandering state. The Ribbon's oscillation amplitude in latitude, about $2^{\circ}$ from peak to trough (Sromovsky et al. 1983), is similar to the width of the jet it resides in, which accompanies a sharp potential vorticity $(\mathrm{PV})$ change over $\sim 5^{\circ}$ in latitude (Read et al. 2009); from this, we infer that the instability is in the regime where nonlinear effects are important.

In this report, we show that the observed wind profile is susceptible to dynamic instabilities through fully nonlinear numerical simulations of Saturn's $47^{\circ} \mathrm{N}$ jet. After the initial linear and nonlinear growth of the eddies, when the background wind speed is fast enough, we demonstrate that the instability nonlinearly saturates and equilibrates at a constant amplitude. When the instability saturates, the $47^{\circ} \mathrm{N}$ jet's morphology, represented by the PV map, resembles that of the cloud patterns observed on Saturn and stably persists over a long time period. Thus, our model explains both the spatial morphology and the longevity of the Ribbon. In addition, we show that the meandering characteristics shown to occur in the Ribbon jet in our model are similar to those of the Gulf Stream in the Atlantic Ocean, which is known for its temporally dynamic meandering path.

On the equatorial (southern) side of the $47^{\circ} \mathrm{N}$ jet is another feature that exhibits longitudinal periodicity, the so-called "string of pearls" (SoPs) discovered in the Cassini Visual and Infrared Mapping Spectrometer (VIMS) 5- $\mu \mathrm{m}$ images (Momary et al. 2006). The "pearls" appear brighter than the surroundings in the $5-\mu \mathrm{m}$ wavelength, suggesting that the feature is a periodic clearing in the clouds (Fig. 3). Choi et al. (2009) measured that the pearls are about $1000 \mathrm{~km}$ in diameter each, spaced around $4^{\circ}$ in longitude and centered at $40^{\circ} \pm 2^{\circ} \mathrm{N}$ latitude. The SoPs share some properties with the $5-\mu \mathrm{m}$ hotspots in the northern low latitudes of Jupiter, which are associated with anticyclonic gyres (Showman and Dowling 2000), although the SoPs are a midlatitude feature and the vorticity of the pearls has not been measured to date. Our simulations of the Ribbon region also produce features that resemble the SoPs and predict that the region is an active source of anticyclonic vortices.

The rest of the report is organized as follows: section 2 presents the setup of our numerical experiments, section 3 discusses our numerical experiments and their results, and section 4 reviews the relevant physical oceanography literature on the meandering ocean currents on Earth and compares them to the observed state of the Ribbon and our modeling results. We present conclusions in section 5 .

\section{Model setup}

\section{a. Numerical model}

We use the Explicit Planetary Isentropic Coordinate (EPIC) model developed by Dowling et al. $(1998,2006)$ to perform our numerical simulations. We use the pure isentropic coordinate version of the model. The simulations presented in this paper employ the following numerical parameters unless otherwise noted. The domain covers the full $360^{\circ}$ in longitude and a channel in latitude spanning $30^{\circ}-60^{\circ} \mathrm{N}$. In the vertical, the top and bottom model layers are placed at pressures around $0.1 \mathrm{hPa}$ $(0.1 \mathrm{mb})$ and $1 \mathrm{MPa}(10 \mathrm{bar})$. The nominal longitude $\times$ latitude $\times$ pressure resolution is $512 \times 64 \times 20$, where the top four layers are the "sponge," preventing artificial reflection of waves at the model top. The 20 layers are evenly spaced in log of pressure (although the model layers are isentropic surfaces, since the initial wind has no vertical shear, the isentropic and isobaric surfaces are parallel and there is no ambiguity in the placement of the model layers initially-the spacing of the layers measured in pressure then evolves with time), which places the top active (i.e., nonsponge) layer at $\sim 1 \mathrm{hPa}$. The sponge relaxes the eastward wind component $u$ to the initial value and the northward component $v$ to zero, which is an implementation of the nonreflection boundary condition at the model top. The lateral boundary conditions are free-slip (i.e., stress-free) at the channel walls to the north and south and periodic to the east and west. The bottom boundary condition is also free-slip. Numerical stability is ensured by the divergence damping component ported from the latest hybrid vertical coordinate version of EPIC, which uses Skamarock and Klemp (1992)'s formulation. The divergence damping coefficient $\nu_{\text {div }}$ is set to $1.62 \times 10^{7} \mathrm{~m}^{2} \mathrm{~s}^{-1}$ or less in all simulations we present here, which represents the lowest 
value necessary to suppress numerical instabilities. We use no hyperviscosity in this study. The time step is $\Delta t=$ $20 \mathrm{~s}$. We tested our results' sensitivities to $\Delta t, \nu_{\text {div }}$, the sponge layer properties, and the model resolution and verified that none of them has a substantial effect.

The values of physical parameters we adopt in our model are the following. The equatorial and polar radii of the planet are $r_{e}=60330 \mathrm{~km}$ and $r_{p}=$ $54180 \mathrm{~km}$, respectively. The gravitational acceleration is set at $8.96 \mathrm{~m} \mathrm{~s}^{-2}$. We use the ideal gas equation of state with the heat capacity with constant pressure $c_{p}=13650 \mathrm{~J} \mathrm{~kg}^{-1} \mathrm{~K}^{-1}$ and specific gas constant $R=$ $3900 \mathrm{~J} \mathrm{~kg}^{-1} \mathrm{~K}^{-1}$. The planetary rotation rate is the commonly accepted System III rate $\Omega=1.6378 \times$ $10^{-4} \mathrm{~s}^{-1}$. Our simulations do not include any radiative or momentum forcing other than the numerical stability terms and the sponge at the model top, and they test the dynamic stability of the zonal jet without any forcing effects.

\section{b. Initial conditions}

Our simulations use the initial zonal wind profiles based on the zonal-mean zonal wind profile measured by Sánchez-Lavega et al. (2000). The initial wind is constant in the vertical; however, as will be discussed later, the wind evolves to acquire a realistic vertical shear in our simulations. As a seed of turbulence, we add spacefilling smooth noise of amplitude $<1 \mathrm{~m} \mathrm{~s}^{-1}$ to the initial wind. The initial noise has a characteristic length scale of $1000 \mathrm{~km}$ and is not in balance with the pressure field unless otherwise noted. We verified that our results are independent of the characteristics of the initial noise when it has an amplitude (length scale) less than or equal to $1 \mathrm{~m} \mathrm{~s}^{-1}(1000 \mathrm{~km})$.

We also test the behavior of the $47^{\circ} \mathrm{N}$ jet under a variety of initial thermal stratifications, since vertical structure is not well constrained in Saturn's troposphere, especially below the cloud tops. Our simulations adopt vertical thermal structures that follow the observed temperature as a function of pressure (e.g., Tyler et al. 1982) in the stratosphere above the $150-\mathrm{hPa}$ level and continuously approach a target Brunt-Väisälä frequency $N$ in the troposphere below the 150 -hPa level. Our synthetic temperature profiles are constructed such that not only the temperature but also $N$ are continuous in the vertical. A discontinuous stratification $N$ can cause unrealistic reflection of waves or act as an artificial waveguide, which can affect the dynamic stability of a zonal jet; accordingly, we keep $N$ continuous in the vertical. Because the tropospheric static stability is not well constrained through observation, we treat it as a free parameter in our study and test our result's sensitivity to the stratification. In the current study, we vary the target

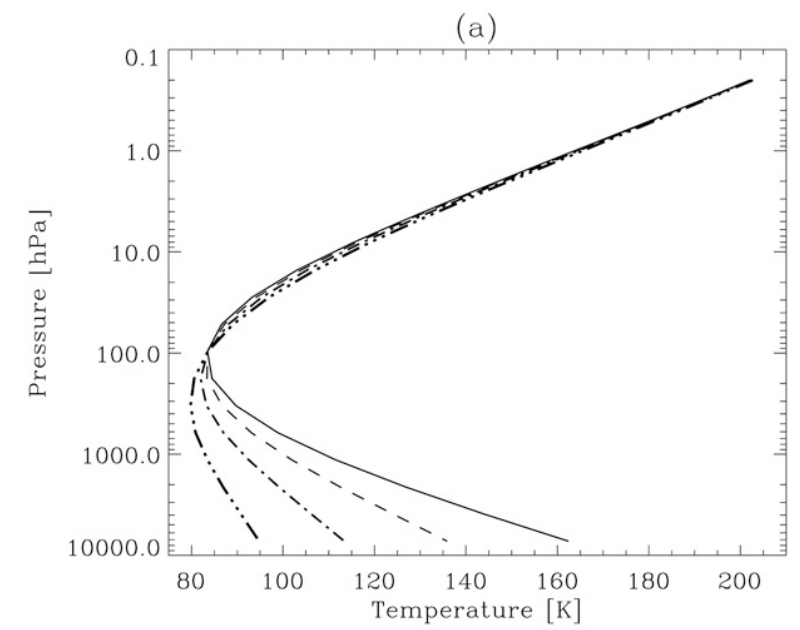

(b)

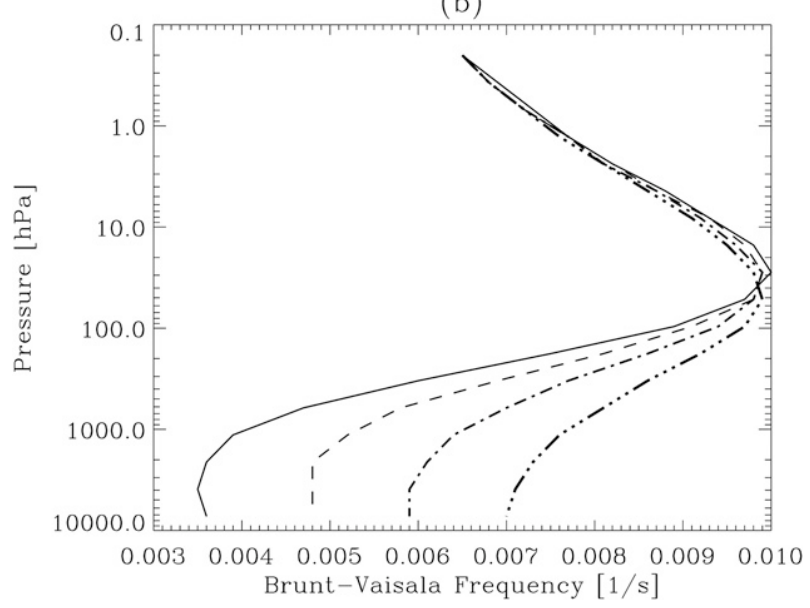

FIG. 4. The vertical thermal profiles used for our simulations(a) temperature vs $p$; (b) $N$ vs $p$. For both, the plotted profiles are $N=0.004$ (solid), 0.005 (dashed), 0.006 (dot-dashed), and $0.007 \mathrm{~s}^{-1}$ (triple dot-dashed).

tropospheric static stability among $N=0.004,0.005$, 0.006 , and $0.007 \mathrm{~s}^{-1}$. We show the vertical temperature and static stability profiles used in our simulations in Figs. 4a,b.

\section{Numerical experiments}

We present the results of our numerical experiments in this section. We first demonstrate that the observed $47^{\circ} \mathrm{N}$ zonal wind profile is susceptible to instabilities. The instability subsequently develops a substantial baroclinic component and then nonlinearly saturates at a steady amplitude without destroying the zonal jet, which naturally evolves into a meandering state. By the time the $47^{\circ} \mathrm{N}$ jet develops a Ribbon-like meandering, the momentum transport due to the instability shapes the jet's vertical shear into a state resembling the observed thermal wind 
TABLE 1. List of simulations.

\begin{tabular}{ccccc}
\hline \hline & $\begin{array}{c}\text { Wind } \\
\text { multiplication } \\
\text { factor }\end{array}$ & $\begin{array}{c}N \\
\left(\mathrm{~s}^{-1}\right)\end{array}$ & $\begin{array}{c}\overline{\boldsymbol{u}} \\
\left(\mathrm{m} \mathrm{s}^{-1}\right)\end{array}$ & $\begin{array}{c}\text { Meandering } \\
\text { phase speed } \\
\left(\mathrm{m} \mathrm{s}^{-1}\right)^{* *}\end{array}$ \\
\hline \multicolumn{2}{l}{ Wind amplitude variation } \\
$\times 0.1 \mathrm{~N} 4$ & 0.1 & 0.004 & 15 & No meandering \\
$\times 0.25 \mathrm{~N} 4$ & 0.25 & 0.004 & 37 & 18 \\
$\times 0.5 \mathrm{~N} 4$ & 0.5 & 0.004 & 74 & 42 \\
$\times 1.0 \mathrm{~N} 4$ & 1.0 & 0.004 & 146 & 80 \\
$\times 1.2 \mathrm{~N} 4$ & 1.2 & 0.004 & 174 & 98 \\
$\times 1.5 \mathrm{~N} 4$ & 1.5 & 0.004 & 217 & 122 \\
$\times 2.0 \mathrm{~N} 4$ & 2.0 & 0.004 & 286 & 162 \\
& & & & \\
Brunt-Väisälä frequency variation & & \\
$\times 1.2 \mathrm{~N} 5$ & 1.2 & 0.005 & 173 & 92 \\
$\times 1.2 \mathrm{~N} 6$ & 1.2 & 0.006 & 171 & 91 \\
$\times 1.2 \mathrm{~N} 7$ & 1.2 & 0.007 & 169 & 87 \\
\hline
\end{tabular}

* The first half of each identifier denotes the wind multiplication factor with respect to the zonal wind measured by SánchezLavega et al. (2000), and the second half denotes the tropospheric $N$. For example, the case $\times 1.2 \mathrm{~N} 6$ has an initial wind profile amplified by a factor of 1.2 , and $N=0.006 \mathrm{~s}^{-1}$.

** The zonal mean wind and meandering phase speed are measured after the simulations reach their nonlinear saturation phase, except for $\times 0.1 \mathrm{~N} 4$ (which did not develop meandering) and $\times 0.25 \mathrm{~N} 4$ (which stayed in the linear growth phase).

shear measured by Read et al. (2009). Second, we test the robustness of our result over a range of background zonal wind speed $\bar{u}(y)$, where $y$ is the north-south distance and the overbar denotes zonal average, and the tropospheric static stability $N$. Through the variation in the background wind profile, we show that the instability occurs when the north-south gradient of PV reverses sign (i.e., when the wind profile violates the Charney-Stern stability criterion). Third, we show that the meanderingjet state can emerge over values of $N$ between 0.004 and $0.007 \mathrm{~s}^{-1}$. The atmospheric static stability is not well known below the clouds on Saturn, and our experiments illustrate that a meandering jet is a stable configuration in a range of conceivable stratification conditions. Finally, we show that a train of vortices reminiscent of SoPs forms through nonlinear instabilities. See Table 1 for the complete list of simulations presented in this report.

\section{a. Development of jet meandering}

We first describe the time evolution of the instability growth using case $\times 1.0 \mathrm{~N} 4$ (see Table 1 ) as an example. Figure 5 illustrates the time evolution of the $47^{\circ} \mathrm{N}$ jet in case $\times 1.0 \mathrm{~N} 4$. The figure shows $\mathrm{PV}$ in grayscale and the thick solid line traces the peak of the $47^{\circ} \mathrm{N}$ jet, defined by the line of zero relative vorticity. The PV plotted here is Ertel's PV defined by

$$
q=\frac{f+\zeta}{h}
$$

where $h$ is the isentropic layer thickness parameter

$$
h=-\frac{1}{g} \frac{\partial p}{\partial \theta} .
$$

Both the PV map and the jet's path are plotted on the isentropic layer placed around the $100-\mathrm{hPa}$ level. In this particular case, in the first $\sim 1000 \mathrm{~h}$ of simulation, the jet's path develops into a sinusoidal line as shown in Fig. 5a, which has roughly 20 peaks spaced irregularly in longitude, captured at hour 480 of the simulation. Fourier decomposing the relative vorticity along $47^{\circ} \mathrm{N}$ latitude as a function of longitude confirms that wavenumber 21 is the single dominant mode. The amplitude of this instability mode grows continuously during the initial phase of instability during which the jet follows a smooth sinusoidal path, which we call the linear growth (LG) phase for the rest of this report. The instability mode has a coherent vertical structure in this phase over most altitudes. Figure 6a shows the longitude-pressure cross section of the deviation of relative vorticity from its zonal mean, defined by $\zeta-\bar{\zeta}$, where $\zeta$ is the relative vorticity. The cross section is made at $47.5^{\circ} \mathrm{N}$ latitude, near the center of the zonal jet at the same instant as in Fig. 5a. The figure shows that the instability's spatial oscillation is largely in phase vertically; this vertical phase coherence characterizes the LG phase.

After approximately hour 800 , the anticyclonic vorticity patches near $46^{\circ} \mathrm{N}$ and the cyclonic patches near $50^{\circ} \mathrm{N}$ start interacting with one another. Figure $5 \mathrm{~b}$ presents the maps of PV and relative vorticity during this phase, which we call the nonlinear growth (NG) phase, shown at hour 1320 of the simulation. As shown in Fig. 5b, the $47^{\circ} \mathrm{N}$ jet path changes from a simple sinusoid to a complex superposition of many wavenumbers with no single wavenumber becoming dominant. Nonlinear effects such as overturning PV contours and merging vorticity patches become prevalent in this phase. The phases of the longitudinal oscillations start to lose their vertical alignment and the amplitude of the eddies becomes substantially greater above $100 \mathrm{hPa}$ than below as shown in Fig. $6 \mathrm{~b}$ at hour 1320 of the simulation. The NG phase lasts until approximately hour 1700 of the simulated time in this case.

The system reaches a statistical equilibrium after $\sim 1700 \mathrm{~h}$, when a balance is reached between the creation and destruction of the vorticity patches to the north and south of the jet core. The vorticity patches are seeded by shear instabilities, and the mechanisms contributing to their destructions are the $\beta$ effect, 
(a) Hour 480

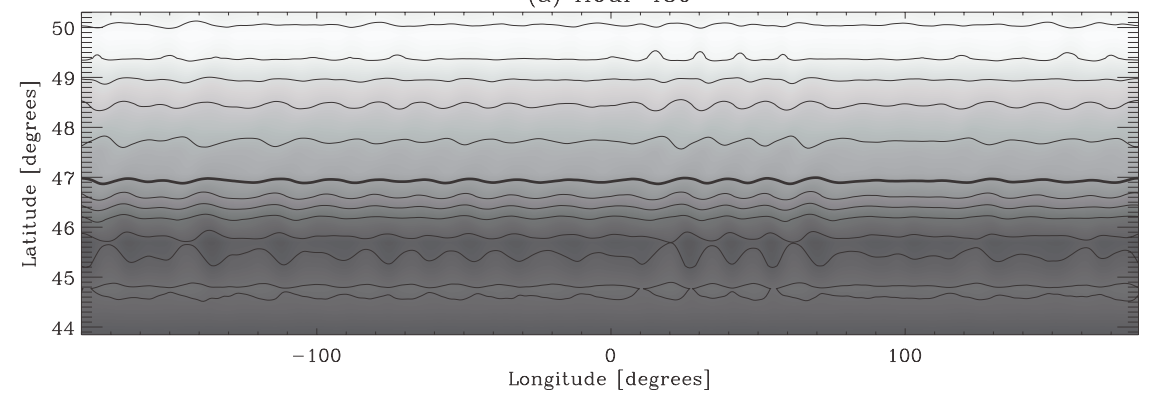

(b) Hour 1320

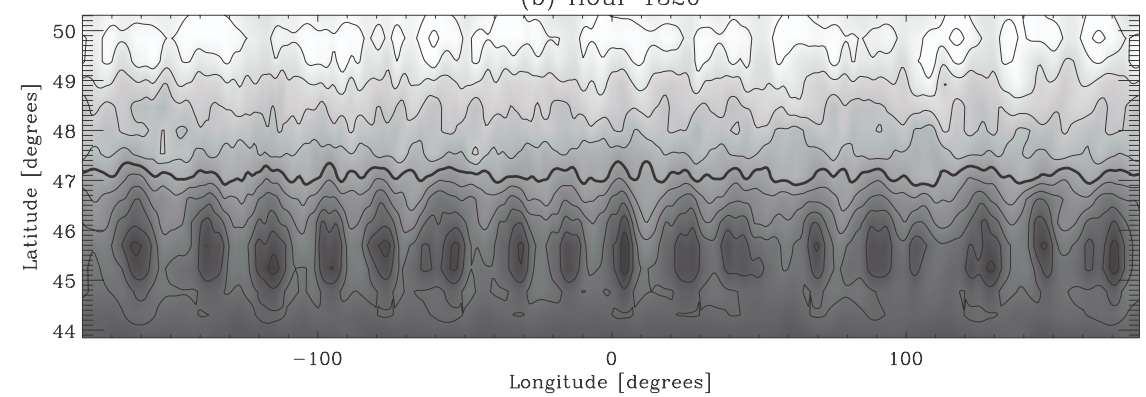

(c) Hour 2400

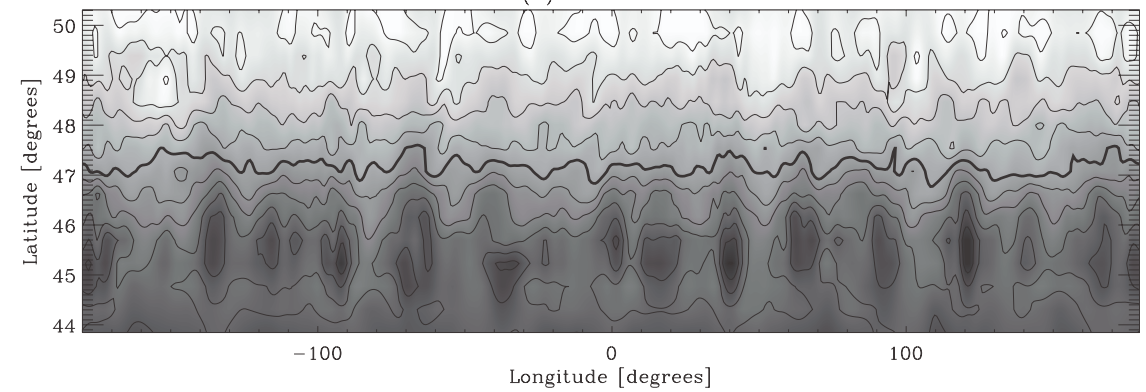

FIG. 5. The grayscale depicts the PV map on the isentropic surface near the 100-hPa level of simulation $\times 1.0 \mathrm{~N} 4$ at three different times: hours (a) 480, (b) 1320, and (c) 2400 . The PV value increases as the grayscale transitions from black to gray to white. The contour lines show the relative vorticity field, with the lines spaced $10^{-5} \mathrm{~s}^{-1}$ apart. The thick line denotes zero relative vorticity, which traces the jet's path.

shearing by the jet, and mergers between multiple vorticity patches. The growth of the jet's meandering amplitude saturates after $\sim 1700 \mathrm{~h}$, and we call this the nonlinear saturation (NS) phase. The jet's morphology develops an irregular zonal pattern with no obvious dominant instability mode as shown in Fig. 5c, and the wind flow continues to exhibit substantial vertical variations as shown in Fig. 6c, depicted at hour 2400 of the simulation.

Once the NS phase is reached, the jet follows a meandering path resembling the Ribbon's morphology. The troughs and crests propagate coherently only for at most several hundred hours, which is illustrated in Fig. 7a-this behavior is hinted at in the Ribbon as shown by Sromovsky et al. (1983). Figure 7a shows the path of the $47^{\circ} \mathrm{N}$ jet every $8 \mathrm{~h}$ between hours 2160 and 2640 of the simulation. The troughs and crests of the jet's meandering move eastward at $\sim 80 \mathrm{~m} \mathrm{~s}^{-1}$ in this case. The figure also shows that the jet occasionally buckles and pinches off a vortex from the jet core, as happens at $140^{\circ} \mathrm{E}$ longitude between hours 2270 and 2320 . This meandering is not dominated by any single wavenumber. In the rest of this report, we identify a jet to be in a chaotic meandering state when no single wavenumber dominates the meandering and the jet exhibits buckling and pinching and spins off vortices. Figure $7 \mathrm{~b}$ shows the Fourier power spectrum of relative vorticity along $47^{\circ} \mathrm{N}$ latitude as a function of planetary wavenumber and time. It illustrates the fluctuating nature of the eddy activities' wavenumber spectrum within the $47^{\circ} \mathrm{N}$ jet and shows that a wavenumber does not stay dominant for more than several hundred hours, which is in agreement with a 

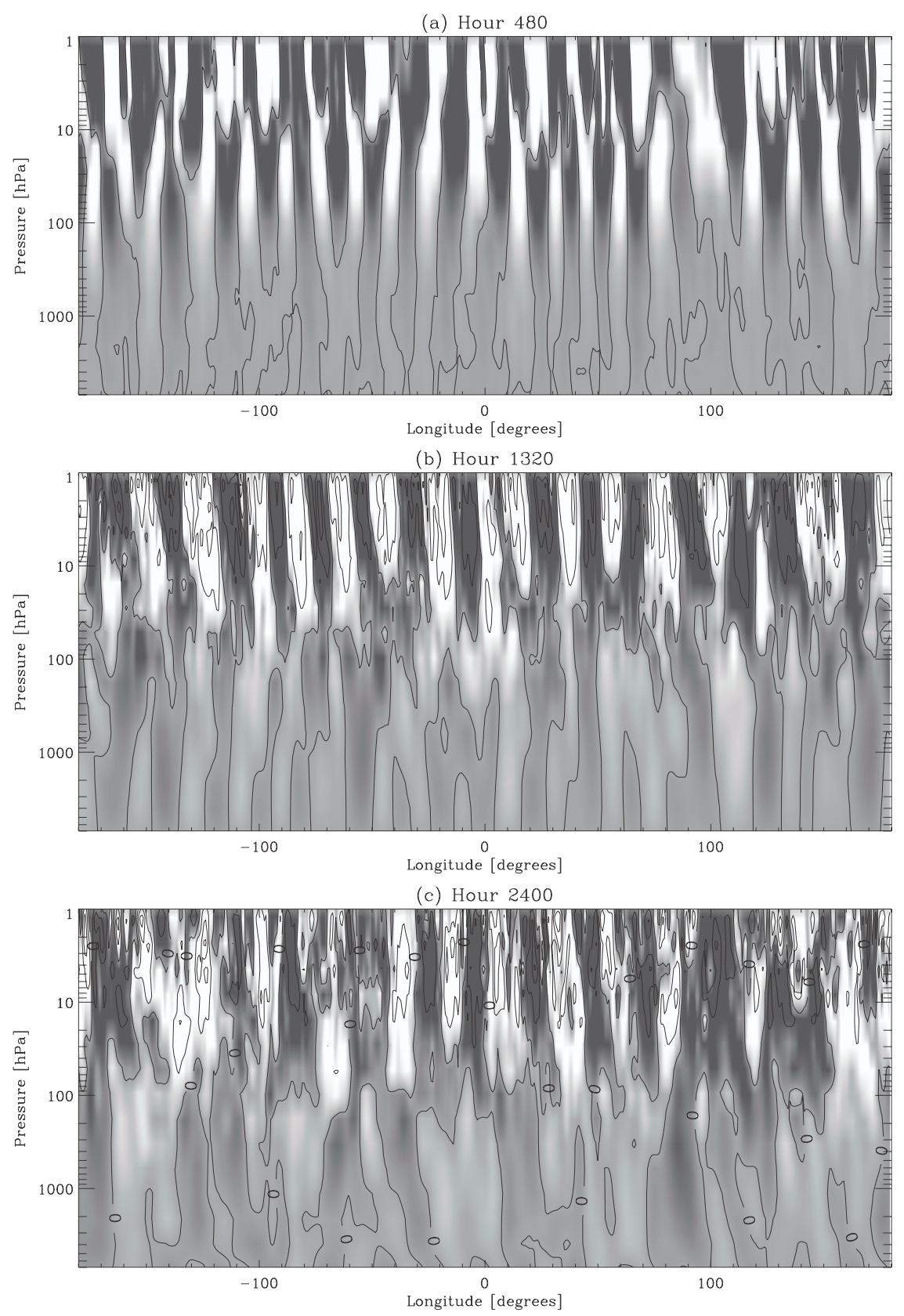

FIG. 6. Longitude $-p$ cross section of $\zeta^{\prime}=\zeta-\bar{\zeta}$ at $47^{\circ} \mathrm{N}$ latitude for case $\times 1.0 \mathrm{~N} 4$. The contour lines are drawn every $10^{-5} \mathrm{~s}^{-1}$. Black and white denote the cyclonic and anticyclonic extrema in the cross section, respectively. Shown are hours (a) 480, (b) 1320, and (c) 2400, the same time steps as in Fig. 2.

typical lifetime of a coherently propagating crests and troughs shown in Fig. 7a.

By the time the Ribbon-like meandering develops, the $47^{\circ} \mathrm{N}$ jet decays in the stratosphere and develops a vertical shear as shown in Fig. 8a, which resembles that derived by Read et al. (2009). Despite the zonal-mean zonal wind speed changes in the stratosphere, the PV profile maintains a sharp gradient, or PV front, around the core of the jet throughout the altitude as shown in Fig. 8b. Figure $8 \mathrm{c}$ shows the zonal standard deviation of the relative vorticity, demonstrating that the eddy amplitude increases with height, as shown in Fig. 6, and the eddies are the most active in the region of the PV front because the instabilities are the most active around the 
(a) Evolution of the Ribbon Path

(b) Evolution of the Spectrum

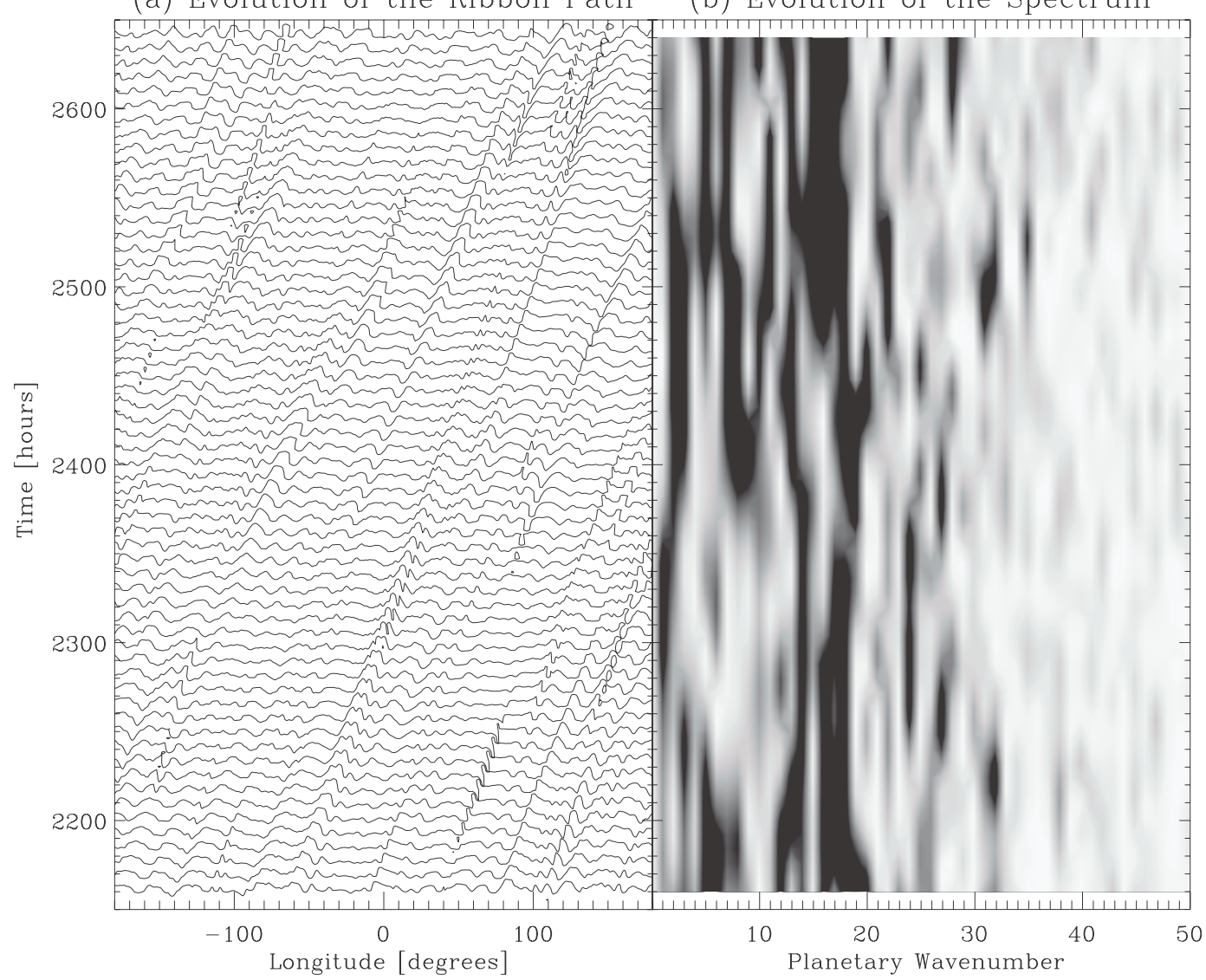

FIG. 7. (a) Time evolution of the $47^{\circ} \mathrm{N}$ jet's meandering path in simulation $\times 1.0 \mathrm{~N} 4$. Each line is a snapshot of the jet's path measured on the isentropic level near $100 \mathrm{hPa}$. A snapshot is made every $8 \mathrm{~h}$ of simulated time between hours 2160 and 2640 of the simulation, and a $1^{\circ}$ latitudinal deviation of the jet's path corresponds to $12 \mathrm{~h}$ on the vertical axis. (b) Time evolution of the Fourier power spectrum of the same simulation during the same time interval. Each pixel denotes the amplitude of a Fourier component at a time step, with black and white indicating the highest and lowest amplitudes, respectively.

points of PV gradient reversals at the north-south extremes of the PV front. At the top of the figure above the $\sim 2$-hPa level, the eddy activities are damped by the sponge layers.

Although the violation of the Charney-Stern stability criterion persists even after the instability saturation, the $47^{\circ} \mathrm{N}$ jet core approaches neutral stability according to Arnol'd's second stability criterion [Arnol'd 1966 (see Arnol'd 1969 for English translation); Dowling 1995] as shown in Fig. 9. Figure 9 shows the gradient of zonal mean pseudo-PV versus $\bar{u}$ for every latitudinal grid point in $\times 1.0 \mathrm{~N} 4$, following the convention in Read et al. (see Figs. 12 and 10 in their 2006 and 2009 papers, respectively). We follow Lebeau and Dowling (1998) to define the pseudo-PV as

$$
q_{G}=q \tilde{h},
$$

where the tilde denotes isentropic layer average such that quantity $A$ is averaged

$$
\tilde{A} \equiv \frac{\iint A d x d y}{\iint d x d y},
$$

where $x$ and $y$ are the eastward and northward distances, respectively. Figures $9 \mathrm{a}$ and $9 \mathrm{~b}$ show the initial condition and hour 4800 of the simulation. Different symbols correspond to different latitude ranges as described in the figure's caption. Note that the diamond points, marking the latitudes between $45^{\circ}$ and $50^{\circ} \mathrm{N}$, approach a linear line in the course of the simulation. Following Read et al. (2006), we write Arnol'd's second criterion as

$$
\frac{\bar{u}-\alpha}{\bar{q}_{G_{y}}} \geqq L_{D}^{2},
$$




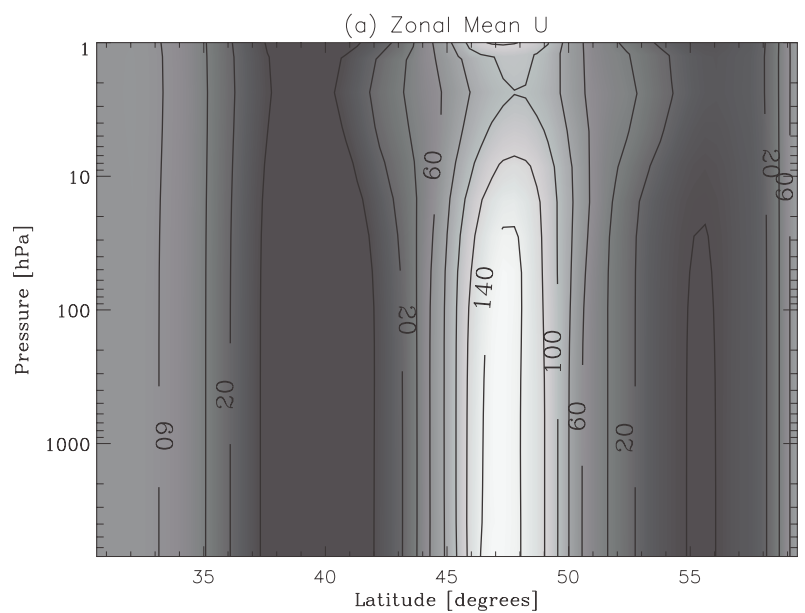

(b) Zonal Mean PV

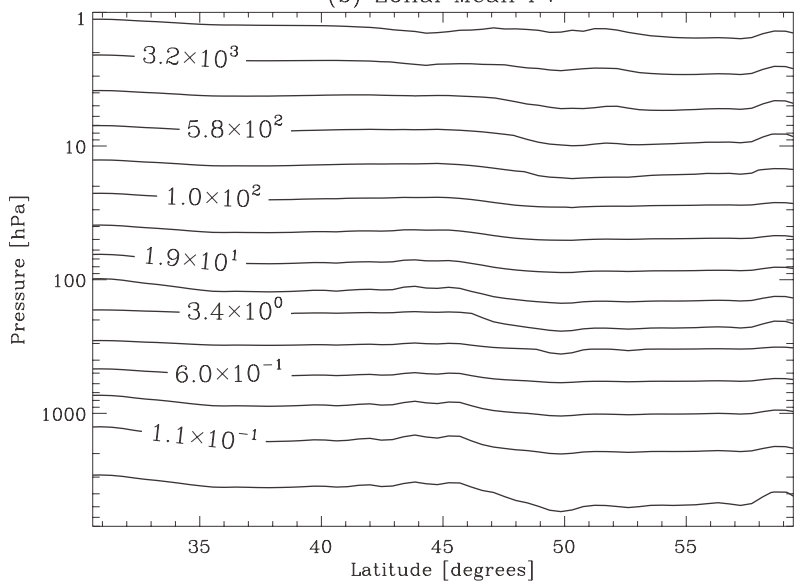

(c) Zonal Stdev of Relative Vorticity

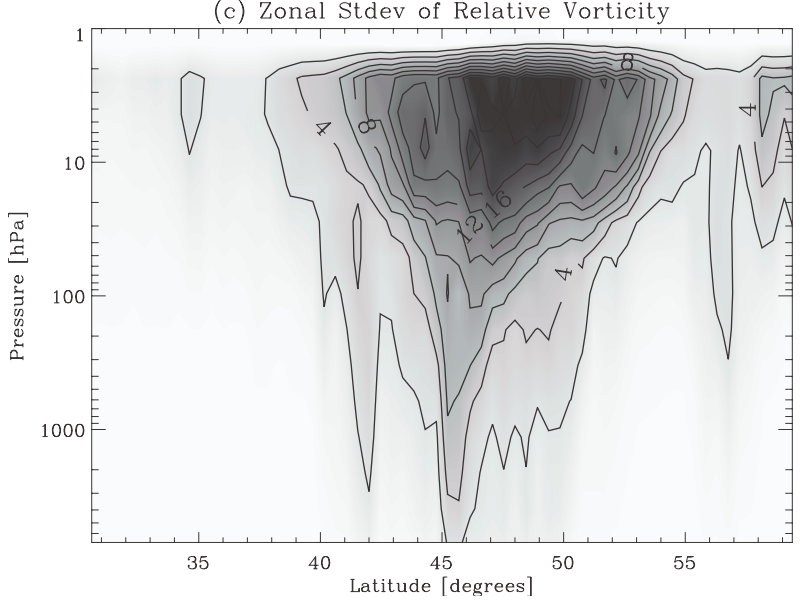

FIG. 8. The zonal mean structure of the $47^{\circ} \mathrm{N}$ jet shown on the latitude- $p$ cross section at hour 2400 of the case $\times 1.0 \mathrm{~N} 4:$ (a) $\bar{u}\left(\mathrm{~m} \mathrm{~s}^{-1}\right)$, (b) zonal mean PV [PV units; 1 PVU $=10^{-6} \mathrm{~K} \mathrm{~m}^{2} \mathrm{~s}^{-1} \mathrm{~kg}^{-1}$ (the contour lines are spaced evenly in log of PV)], and (c) the zonal standard deviation of relative vorticity $\zeta_{\mathrm{SD}}=\left(\bar{\zeta}^{2}-\bar{\zeta}^{2}\right)^{1 / 2}\left(10^{-5} \mathrm{~s}^{-1}\right)$. (a) Hour 0

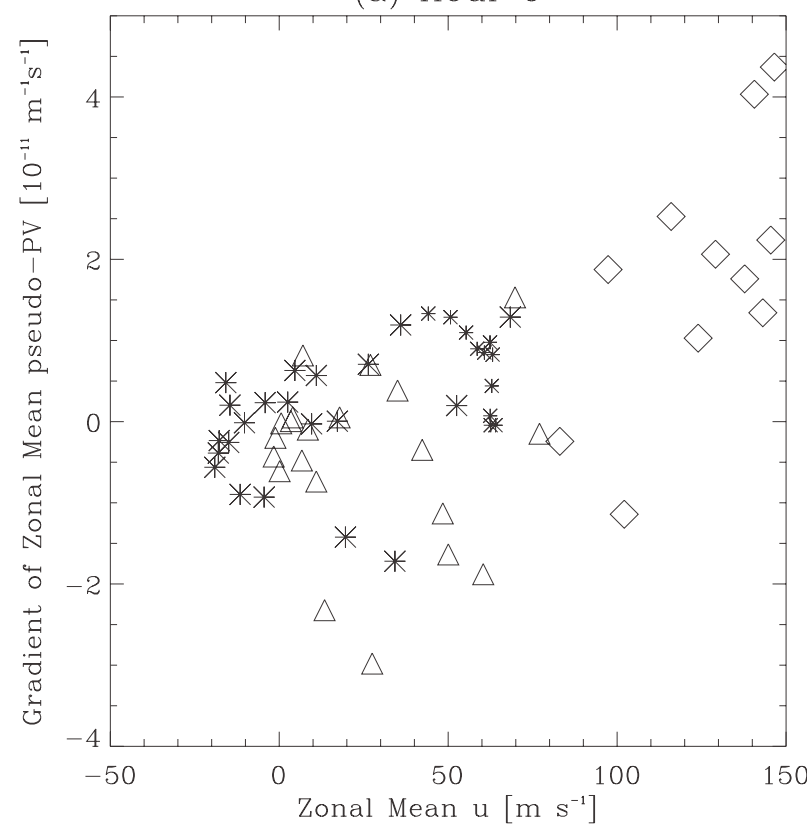

(b) Hour 4800

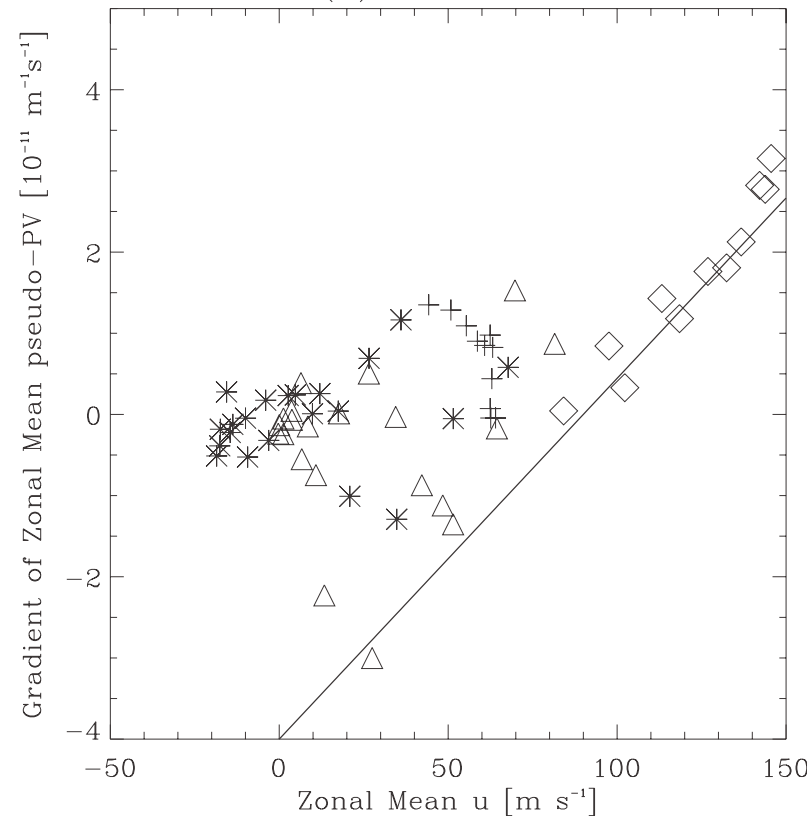

FIG. 9. The vertical axis is $\bar{q}_{G}$, the horizontal axis is $\bar{u}$, and each data point represents a latitudinal grid. The symbols denote different latitude range; the pluses, asterisks, diamonds, and triangles denote $30^{\circ}-35^{\circ} \mathrm{N}, 35^{\circ}-45^{\circ} \mathrm{N}, 45^{\circ}-50^{\circ} \mathrm{N}$, and $50^{\circ}-60^{\circ} \mathrm{N}$, respectively, in both (a) the initial condition and (b) after $4800 \mathrm{~h}$ of simulation. The straight line in (b) denotes a reference slope with $L_{D}=1500 \mathrm{~km}$.

where $\bar{q}_{G_{y}}=\partial \bar{q}_{G} / \partial y$ and $\alpha$ is a reference frame shift speed. The neutral stability occurs when the two sides of (5) are equal. Thus, under neutral stability, $\bar{q}_{G_{y}}$ becomes a linear function of $\bar{u}$ with slope $L_{D}^{-2}$. Figure $9 \mathrm{~b}$ shows 
a reference line with $L_{D}=1500 \mathrm{~km}$ to illustrate that the diamond points closely follow the reference slope, where $L_{D}=1500 \mathrm{~km}$ is similar to that measured by Read et al. (2009) for Saturn's $47^{\circ} \mathrm{N}$ jet. Interestingly, the diamond points suggest $\alpha \sim 90 \mathrm{~m} \mathrm{~s}^{-1}$, which is close to the propagation speed of the meandering, $80 \mathrm{~m} \mathrm{~s}^{-1}$, as shown in Fig. 7. Note that $\alpha$ is generally interpreted as the propagation speed of the most unstable mode, so the match between $\alpha$ and the meandering propagation speed here is intriguing.

The temporal evolution of the kinetic energy components further characterizes the instability's growth. The total kinetic energy of the system is

$$
K_{\text {Total }}=\int \frac{h}{2}\left(u^{2}+v^{2}\right) d V,
$$

where $d V \equiv d x d y d \theta$ is the differential volume element in the isentropic coordinate ( $\theta$ is the potential temperature). First, we introduce the mass-weighted averaging operators in the zonal and vertical dimensions. Similar to Peixoto and Oort (1992)'s notations, we define the zonal averaging by

$$
[A] \equiv \frac{\int A h d x}{\int h d x}
$$

and the vertical averaging by

$$
\langle A\rangle \equiv \frac{\int A h d \theta}{\int h d \theta} .
$$

The deviations from the zonal and vertical averages are defined as

$$
A^{*} \equiv A-[A]
$$

and

$$
A^{\prime \prime} \equiv A-\langle A\rangle,
$$

respectively. Using the above, we define the zonal barotropic (ZBT), zonal baroclinic (ZBC), eddy barotropic (EBT), and eddy baroclinic (EBC) components of the kinetic energy as

$$
\begin{aligned}
K_{\mathrm{ZBT}} & =\int \frac{h}{2}\left(\langle[u]\rangle^{2}+\langle[v]\rangle^{2}\right) d V, \\
K_{\mathrm{ZBC}} & =\int \frac{h}{2}\left([u]^{\prime \prime 2}+[v]^{\prime \prime 2}\right) d V,
\end{aligned}
$$

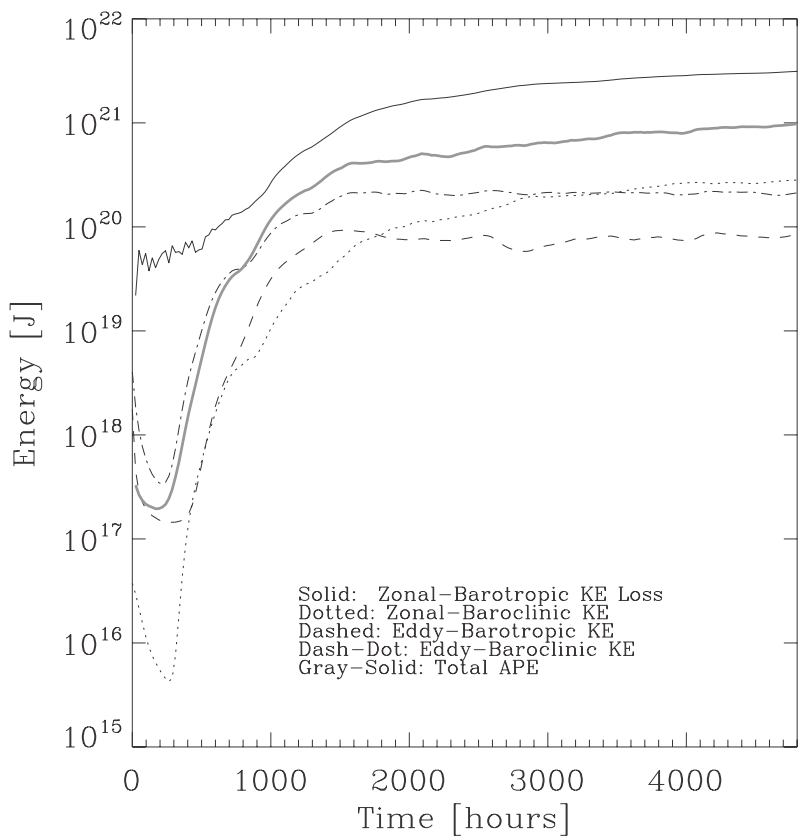

FIG. 10. The time evolution of kinetic energy components $K_{\mathrm{ZBT}}$, $K_{\mathrm{ZBC}}, K_{\mathrm{EBT}}$, and $K_{\mathrm{EBC}}$, and APE for the case $\times 1.0 \mathrm{~N} 4$. The quantities are defined in section $3 \mathrm{a}$. Because the zonal barotropic component is much greater than the others, its loss $K_{\mathrm{ZBT}}-K_{\mathrm{ZBT}}(t=0)$ is shown instead.

$$
K_{\mathrm{EBT}}=\int \frac{h}{2}\left(\left\langle u^{*}\right\rangle^{2}+\left\langle v^{*}\right\rangle^{2}\right) d V,
$$

and

$$
K_{\mathrm{EBC}}=\int \frac{h}{2}\left\{\left(u^{*}\right)^{\prime \prime 2}+\left(v^{*}\right)^{\prime \prime 2}\right\} d V
$$

respectively. Consequently, the zonal and eddy components of the kinetic energy are

$$
K_{\text {Zonal }}=K_{\text {ZBT }}+K_{\text {ZBC }}
$$

and

$$
K_{\mathrm{Eddy}}=K_{\mathrm{EBT}}+K_{\mathrm{EBC}} \cdot
$$

Note that above formulation keeps $K_{\text {Total }}=K_{\text {Zonal }}+$ $K_{\text {Eddy }}$ exact, unlike the formulation employed by Sayanagi et al. (2008), who discuss that their zonal-eddy decomposition is only approximate due to the noncancelation of the "cross terms."

We show the time evolution of the four kinetic energy components for the nominal case $\times 1.0 \mathrm{~N} 4$ in Fig. 10 . As the zonal-barotropic component is many orders of magnitude greater than the other three, we present its loss $K_{\mathrm{ZBT}}(t=0)-K_{\mathrm{ZBT}}$. In Fig. 10 , the $K_{\mathrm{ZBT}}$ loss fluctuates 

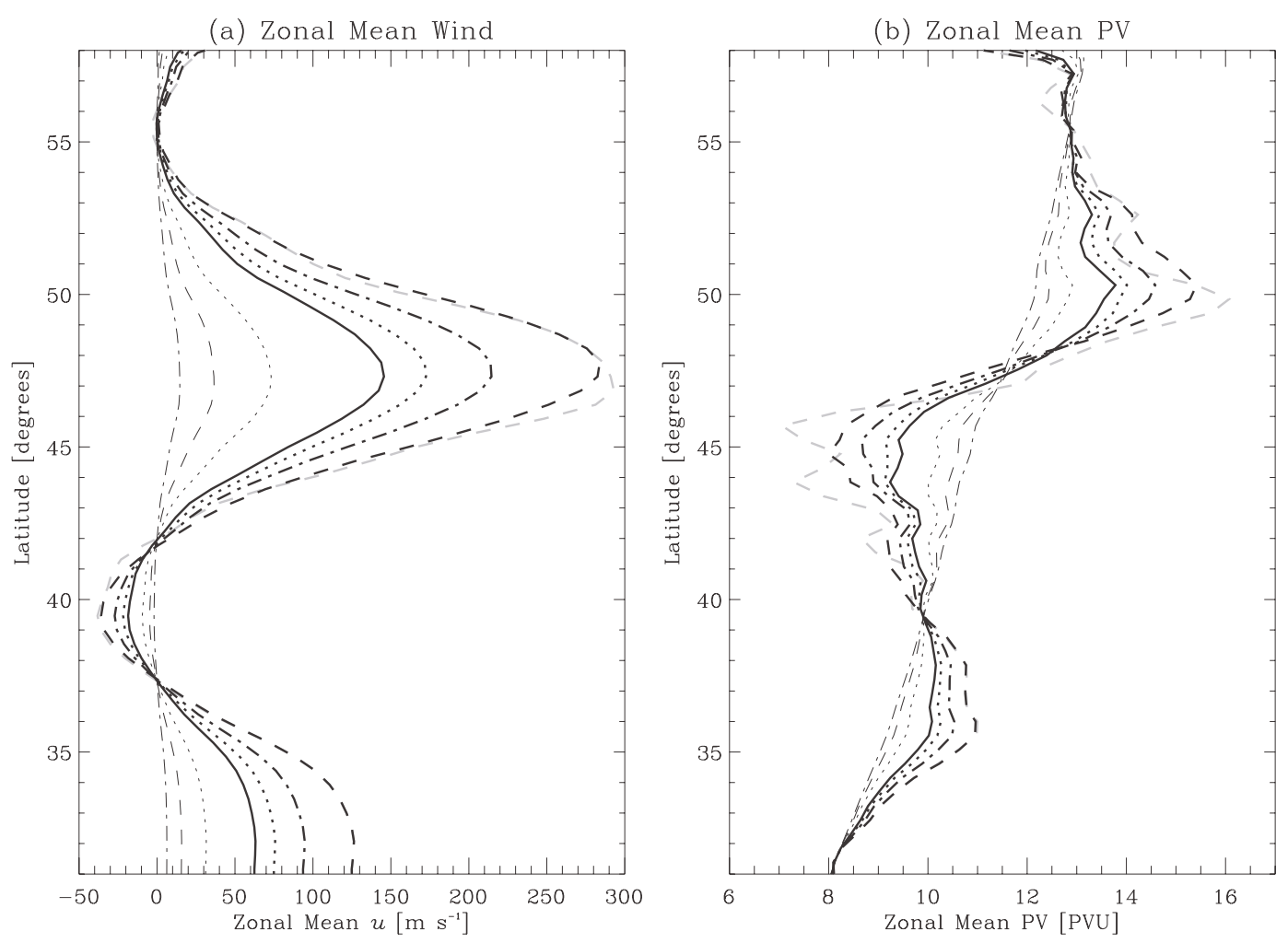

FIG. 11. Zonal mean profiles of (a) $u$ and (b) PV, for simulations $\times 0.1 \mathrm{~N} 4$ (thin dot-dashed), $\times 0.25 \mathrm{~N} 4$ (thin dashed), $\times 0.5 \mathrm{~N} 4$ (thin dotted), $\times 1.0 \mathrm{~N} 4$ (solid), $\times 1.2 \mathrm{~N} 4$ (thick dotted), $\times 1.5 \mathrm{~N} 4$ (thick dot-dashed), and $\times 2.0 \mathrm{~N} 4$ (thick dashed).

The thick gray dashed lines denote the initial profiles for the case $\times 2.0$ N4. In (b), the horizontal axis is in PVU.

in the first $\sim 600 \mathrm{~h}$ because of truncation errors resulting from taking a difference between two large numbers. As the figure shows, $K_{\mathrm{ZBC}}, K_{\mathrm{EBT}}$, and $K_{\mathrm{EBC}}$ initially decay as the unbalanced initial small perturbations excite shortwavelength gravity waves, which are quickly dissipated. After $\sim 300 \mathrm{~h}, K_{\mathrm{ZBC}}, K_{\mathrm{EBT}}$, and $K_{\mathrm{EBC}}$ undergo rapid growth during the LG phase up to $\sim 800 \mathrm{~h}$. After hour $\sim 800$, their growth rates slightly decreases, marking the NG phase. The eddy and baroclinic kinetic energy components plateau after $\sim 1700 \mathrm{~h}$ when the system reaches the NS phase. As shown in Figs. 6 and 8c, the amplitude of the eddies grows with altitude; however, because the mass density decreases with altitude, only $\sim 25 \%$ of the eddy kinetic energy is located above $10 \mathrm{hPa}$.

Figure 10 also shows the temporal evolution of the total available potential energy (APE) of the system. We calculate APE using Lorenz (1955)'s formulation,

$$
\mathrm{APE}=\frac{c_{p}}{g(1+\kappa) p_{0}^{\kappa}} \int\left(p^{\kappa+1}-\tilde{p}^{\kappa+1}\right) d V
$$

where $p$ is pressure, $p_{0}=1000 \mathrm{hPa}$ is the reference pressure, and $\kappa=R / c_{p}$. The figure demonstrates that as the winds' baroclinic components grow to substantial amplitudes, the isentropic surfaces tilt, resulting in the evolution of APE similar to the growth and plateauing of the eddy and baroclinic kinetic energy components.

\section{b. Background wind amplitude variation}

Our nominal case illustrates that in forming a meandering jet with a Ribbon-like morphology, nonlinear wave effects at the flanks of the $47^{\circ} \mathrm{N}$ jet, including waveinduced overturning of the PV gradient, play an important role. Past studies (e.g., Sayanagi and Showman 2007) show that the background PV gradient associated with an eastward zonal jet such as the $47^{\circ} \mathrm{N}$ jet has a substantial impact on the wave-induced PV overturning process. Therefore, to study the effect of PV gradient on the meandering instability, we change the zonal wind profile by a constant multiplication factor.

For this experiment, we scale the wind measured by Sánchez-Lavega et al. (2000) by multiplication factors $2.0,1.5,1.2,0.5,0.25$ and 0.1 (cases $\times 2.0 \mathrm{~N} 4, \times 1.5 \mathrm{~N} 4$, $\times 1.2 \mathrm{~N} 4, \times 0.5 \mathrm{~N} 4, \times 0.25 \mathrm{~N} 4$, and $\times 0.1 \mathrm{~N} 4$, respectively) and compare their results to the nominal case $\times 1.0 \mathrm{~N} 4$. Figure 11a shows their zonal mean wind profiles after these cases reach their respective NS phases. As exemplified by case $\times 2.0 \mathrm{~N} 4$ in the figure (the thick dashed lines; 
gray indicates the initial condition and black the NS phase), the change of wind profiles in each of these cases in the course of the simulations is relatively minor below the 100 -hPa level, with the $47^{\circ} \mathrm{N}$ jet losing its peak speed at the $100-\mathrm{hPa}$ level by $\sim 7 \%$. Above $100 \mathrm{hPa}$, the winds decay substantially as in the nominal case, and the faster-wind cases exhibit greater fractional speed changes. Figure $11 \mathrm{~b}$ compares the end-state zonal PV profiles of the simulations after the instabilities are allowed to develop. Changes to the zonal mean PV profiles during the course of the simulations are minor. The comparison between the initial and the equilibrated PV profiles of $\times 2.0 \mathrm{~N} 4$ reveals minor homogenization of the PV but the overall structure remains largely intact throughout the instability process. Also, when simulations reach the NS phase, the PV map exhibits substantial zonal scatter around the latitude where the PV gradient switches sign (e.g., around $45^{\circ}$ and $50^{\circ} \mathrm{N}$ ). The zonal PV scatter is also greater between those two latitudes, where the meandering instability is active, than outside.

Figure $11 \mathrm{~b}$ illustrates that $\times 0.1 \mathrm{~N} 4$ has a monotonically increasing PV as a function of latitude, and $\times 0.25 \mathrm{~N} 4$ has regions of approximately constant $\mathrm{PV}$ between $35^{\circ}$ and $46^{\circ} \mathrm{N}$ and to the north of $50^{\circ} \mathrm{N}$. The cases with higheramplitude wind-scaling factors have gradually steeper PV gradients and reversals of PV gradient at $45^{\circ}$ and $50^{\circ} \mathrm{N}$ latitudes, both associated with the $47^{\circ} \mathrm{N}$ jet.

Figure 12 shows the maps of PV and relative vorticity for cases $\times 0.25 \mathrm{~N} 4, \times 0.5 \mathrm{~N} 4, \times 1.5 \mathrm{~N} 4$, and $\times 2.0 \mathrm{~N} 4$ after each simulation reached its respective NS phase, except for $\times 0.25 \mathrm{~N} 4$, which did not produce chaotic meandering. These simulations illustrate that the meandering occurs when the $47^{\circ} \mathrm{N}$ jet causes reversals in the north-south gradient of PV (i.e., when the jet violates the CharneyStern stability criterion). The weakest-wind case that developed instability, $\times 0.25 \mathrm{~N} 4$, which barely causes reversals in the north-south PV gradient, did not develop chaotic meandering as shown in Fig. 12a at the end of its 24000 -h-long run although the amplitude of the instability did reach saturation. The jet's path in this case exhibits characteristics similar to those of $\times 1.0 \mathrm{~N} 4$ during its LG phase, including a simple sinusoidal path with no overturning of PV gradient. Case $\times 0.5 \mathrm{~N} 4$ (Fig. 12b) reaches the NS phase exhibiting chaotic meandering behaviors including buckling of the jet path and PV overturning, although the frequency of the buckling and the amplitude of its latitudinal diversion are much smaller than in the faster-wind cases. Also, the vorticity patches to the north and south of the $47^{\circ} \mathrm{N}$ jet do not become as prominent as in the stronger-wind cases. In contrast, in $\times 1.5 \mathrm{~N} 4$ (Fig. $12 \mathrm{c}$ ) and $\times 2.0 \mathrm{~N} 4$ (Fig. $12 \mathrm{~d}$ ), the vorticity patches become pronounced and affect the meandering of the $47^{\circ} \mathrm{N}$ jet substantially. In $\times 1.5 \mathrm{~N} 4$ and $\times 2.0 \mathrm{~N} 4$, the $47^{\circ} \mathrm{N}$ jet path follows the perimeters of these vorticity patches, and the meandering becomes dominated by a stable wavenumber determined by the number of vorticity patches that emerge. The large-amplitude meandering caused by these vorticity patches propagates coherently for the remainder of the simulations, which we run for at least $3000 \mathrm{~h}$ after the onset of the NS phase. The coherent propagation of the meandering and the large vorticity patches associated with the high-amplitude wind simulations $\times 1.5 \mathrm{~N} 4$ and $\times 2.0 \mathrm{~N} 4$ are not very similar to the behaviors of the Ribbon noted by Sromovsky et al. (1983).

The time evolution of $K_{\text {Eddy }}$ further illustrates the eddy growths in these simulations, shown in Fig. 13, which shows that $K_{\text {Eddy }}$ grows faster and to greater magnitudes in cases with faster background jets, and hence a steeper PV gradient. We do not estimate the linear growth rates in their LG phases, however, because the exponential growth rates are not constant, as evident in Fig. 13a; the $K_{\text {Eddy }}$ curves are not straight lines in this log-axis plot. Note that the initial seed of turbulence has the same absolute amplitude in all simulations except $\times 1.2 \mathrm{~N} 4$, which has initial perturbations twice as large. The initial perturbations in case $\times 1.2 \mathrm{~N} 4$ are also in gradient balance and do not excite gravity waves, thus its $K_{\text {Eddy }}$ does not decay initially. Despite the stronger, balanced initial perturbations, $\times 1.2 \mathrm{~N} 4$ 's final $K_{\text {Eddy }}$ amplitude still fits the trend among the cases with weaker initial perturbation, demonstrating that the instability's development depends more strongly on the jet profile than the initial perturbation. Figure 13b shows the same quantity as in Fig. 13a over a longer time scale, which illustrates that the instability saturates in case $\times 0.5 \mathrm{~N} 4$ after $\sim 4000 \mathrm{~h}$ of simulation whereas case $\times 0.25 \mathrm{~N} 4$ exhibits a slow asymptotic increase, which continues at least up to the end of the 24000 h-long simulation.

\section{c. Effects of tropospheric static stability}

In addition to the initial wind speeds, we varied the tropospheric $N$ as presented in section $2 \mathrm{~b}$. The results are consistent with the wind speed variation experiment. As noted by (1), higher $N$ reduces $h$ and thus amplifies $\mathrm{PV}$ and its gradient. Figure 14 illustrates this point, which shows the zonal mean PV profiles of simulations $\times 1.2 \mathrm{~N} 4$, $\times 1.2 \mathrm{~N} 5, \times 1.2 \mathrm{~N} 6$, and $\times 1.2 \mathrm{~N} 7$ at the end of each simulation (hour 4800), which is well after the onset of the NS phase for each case. As in the earlier series of simulations, the temporal changes to the zonal mean PV profile throughout the course of simulations are minor. The figure shows that the cases with greater $N$ have slightly larger PV changes across the $47^{\circ} \mathrm{N}$ jet, between $45^{\circ}$ and $50^{\circ} \mathrm{N}$.

Figure 15 shows $K_{\text {Eddy }}$ for the simulations in the $N$ variation experiment. The figure shows a trend that, as in 



FIG. 12. PV maps of simulations (a) $\times 0.25 \mathrm{~N} 4$, (b) $\times 0.5 \mathrm{~N} 4$, (c) $\times 1.5 \mathrm{~N} 4$, and (d) $\times 2.0 \mathrm{~N} 4$ on the model layer near $100 \mathrm{hPa}$ after instabilities are allowed to develop in each of the simulations. Black and white denote the lowest and highest values of PV, respectively. The contour lines show the relative vorticity field; the contour line spacing is $0.25 \times 10^{-5} \mathrm{~s}^{-1}$ in (a) and (b) and $10^{-5} \mathrm{~s}^{-1}$ in (c) and (d). The thick contour lines note zero relative vorticity.

the wind speed variation experiment, cases with larger PV change across the zonal jet tends to exhibit faster instability growth and $K_{\text {Eddy }}$ saturates at higher values. Although the difference between the $N=0.006 \mathrm{~s}^{-1}$ and the $N=0.007 \mathrm{~s}^{-1}$ cases $(\times 1.2 \mathrm{~N} 6$ and $\times 1.2 \mathrm{~N} 7$, respectively) is minor, $K_{\text {Eddy }}$ mostly stays greater in the higher- $N$ case. Cases with higher $N$, and thus a bigger change in $\mathrm{PV}$ across the $47^{\circ} \mathrm{N}$ jet, also develop more pronounced vorticity patches associated with the enhanced eddy activities, which is also consistent with the wind speed variation experiment. All cases presented in this panel had initial perturbations that were balanced and they do not 


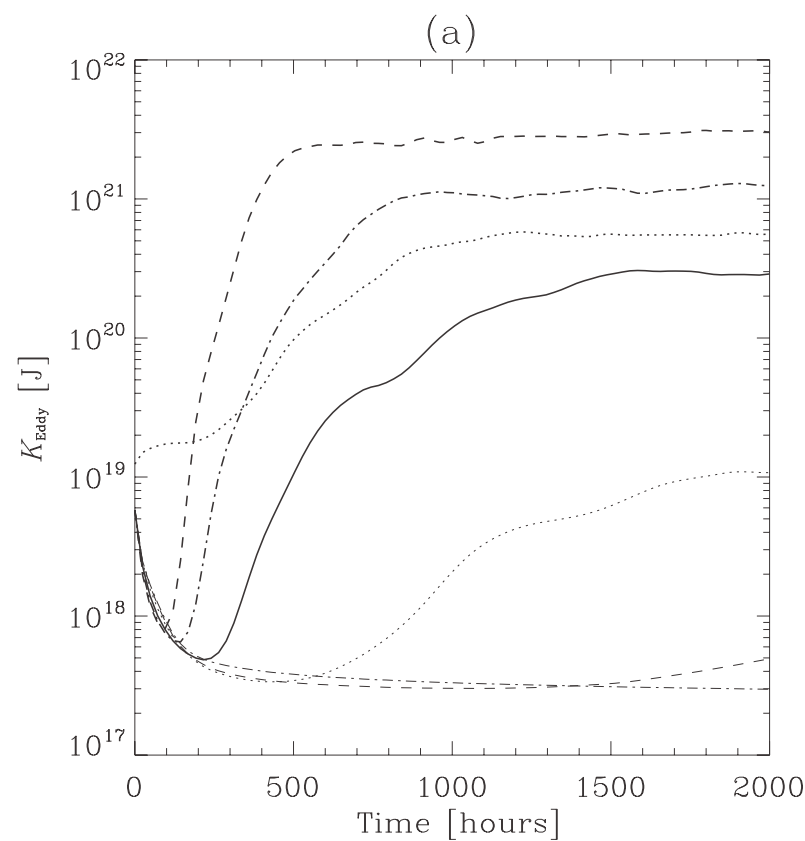

(b)

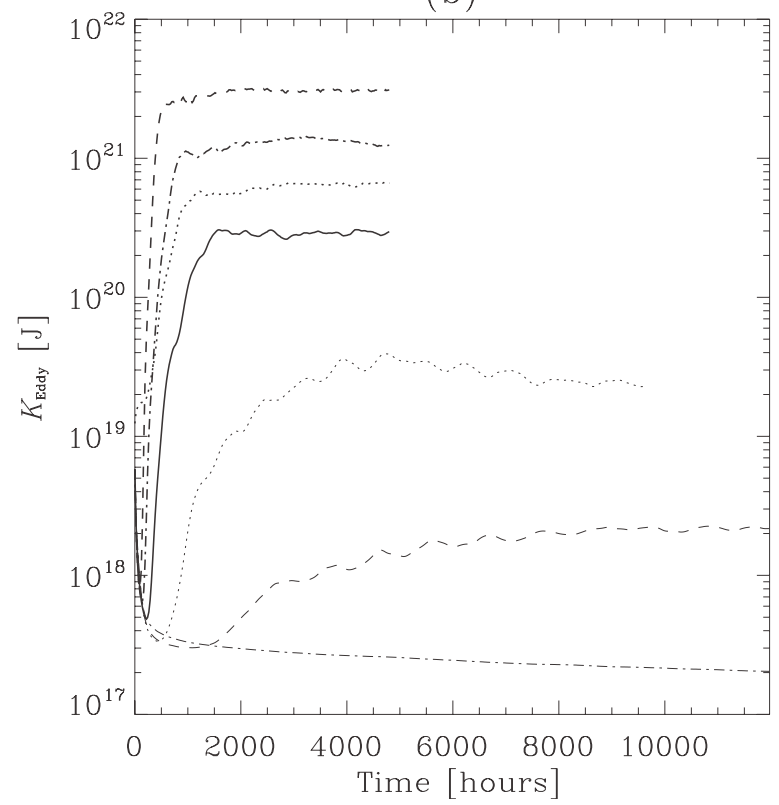

FIG. 13. The time evolution of $K_{\text {Eddy }}$ of the same set of simulations shown in Fig. 10, with the same line styles corresponding to the same simulations, using (a) only the first $2000 \mathrm{~h}$ of the simulations (to illustrate the initial growth) and (b) longer time scales (to show the longer-term trends of the same quantities).

exhibit the initial drop in $K_{\text {Eddy }}$, unlike the cases with unbalanced initial perturbations.

\section{d. Note on the background wind speed and the meandering phase speed}

As noted in the introduction, the observed phase speed of the Ribbon propagation and the local peak

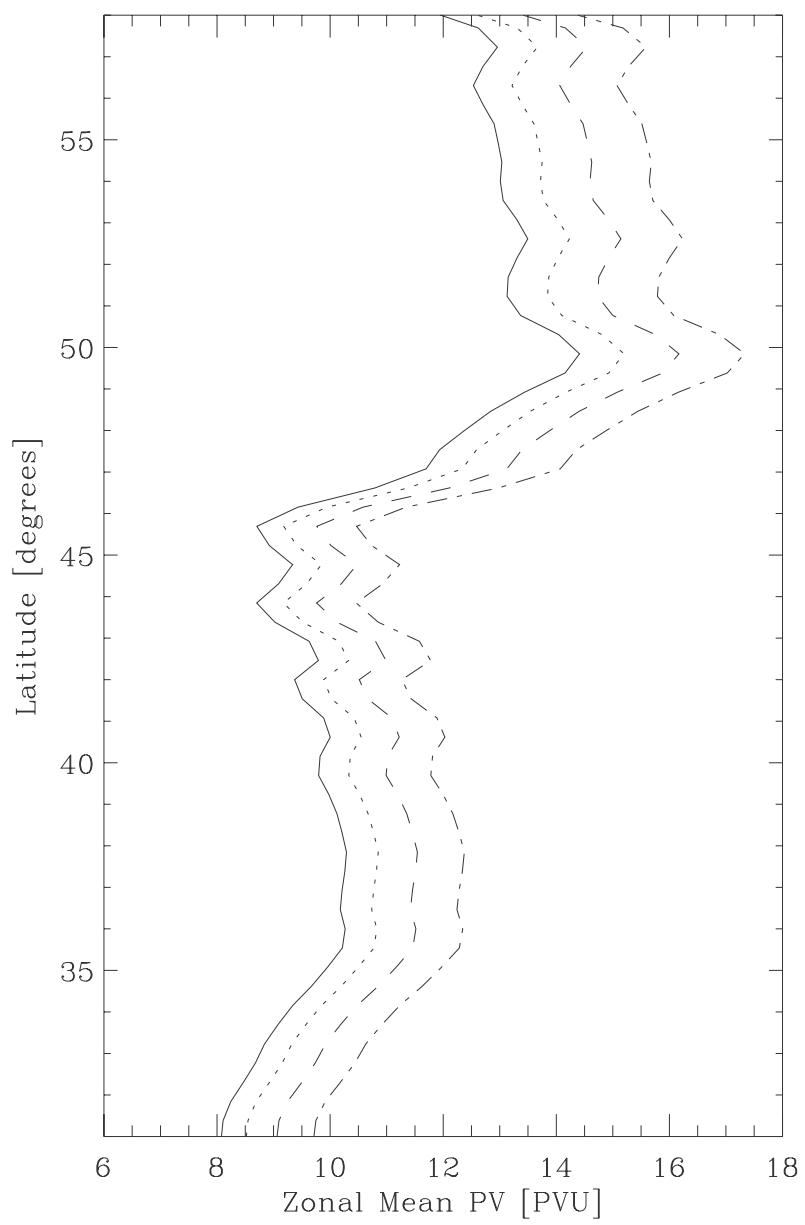

FIG. 14. Zonal mean PV profiles of $\times 1.2 \mathrm{~N} 4$ (solid), $\times 1.2 \mathrm{~N} 5$ (dotted), $\times 1.2 \mathrm{~N} 6$ (dashed), and $\times 1.2 \mathrm{~N} 7$ (dot-dashed) after the instabilities reach the NS phase in each of the simulations.

zonal jet speed match at $\sim 150 \mathrm{~m} \mathrm{~s}^{-1}$, which we suspect may be caused by contamination of cloud tracking measurement by wave activities in the Ribbon region. In our experiment, we treat the background wind speed as a free parameter to explore the conditions that allow the Ribbon to propagate at $\sim 150 \mathrm{~m} \mathrm{~s}^{-1}$. We also vary the tropospheric static stability $N$ as done in the aforementioned series of simulations and analyzed its effect on the phase speed of the meandering. The results are summarized in Table 1. As shown in the table, we found that varying $N$ does not have significant effects on the meander phase speed. In the cases that develop the NS phase, the meanders propagate at a speed approximately $60 \%$ slower than the peak zonal mean wind speed of the $47^{\circ} \mathrm{N}$ jet. Our simulations indicate that for the Ribbon to have $150 \mathrm{~m} \mathrm{~s}^{-1}$ propagation phase speed, the background wind speed should be approximately $250 \mathrm{~m} \mathrm{~s}^{-1}$, which is substantially faster than $\sim 200 \mathrm{~m} \mathrm{~s}^{-1}$, the speed of the fastest-moving cloud 


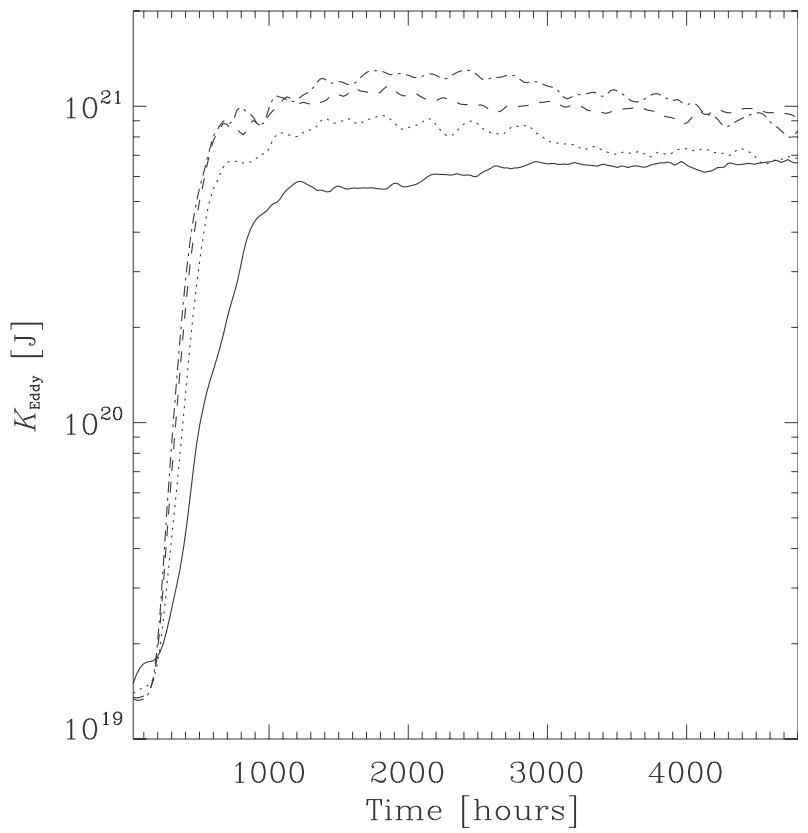

FIG. 15. Temporal evolution of $K_{\text {Eddy }}$ for simulations $\times 1.2 \mathrm{~N} 4$ (solid), $\times 1.2 \mathrm{~N} 5$ (dotted), $\times 1.2 \mathrm{~N} 6$ (dashed), and $\times 1.2 \mathrm{~N} 7$ (dotdashed).

in the $47^{\circ} \mathrm{N}$ jet found by Sánchez-Lavega et al. (2000, see their Fig. 2a).

\section{e. Vortex formations at $41.5^{\circ} \mathrm{N}$}

In addition to the meandering jet, our simulations serendipitously produced a chain of anticyclonic vortices centered at $41.5^{\circ} \mathrm{N}$ latitude at the equatorward (southern) edge of the $47^{\circ} \mathrm{N}$ jet as shown in Fig. 16, which shows the relative vorticity of the case $\times 1.0 \mathrm{~N} 4$ in the entire horizontal simulation domain on the isentropic layer corresponding to the $100-\mathrm{hPa}$ level. The vortices form as a result of the instability at the trough (i.e., westward peak) of the zonal wind profile, where the curvature of the zonal wind profile (i.e., $\partial^{2} \bar{u} / \partial y^{2}$ ) has a local maximum, and consequently, the barotropic stability criterion

$$
\beta-\frac{\partial^{2}}{\partial y^{2}} \bar{u}>0
$$

is most strongly violated in the simulation domain. (Here, we refer to the barotropic criterion simply to note the latitude of maximum wind curvature. For predicting the flow's stability, the Charney-Stern criterion is more relevant for our simulations, in which violations occur at 45 and $50^{\circ} \mathrm{N}$ latitudes as discussed in section $3 \mathrm{~b}$.) Our simulations $\times 0.5 \mathrm{~N} 4, \times 1.2 \mathrm{~N} 4, \times 1.5 \mathrm{~N} 4$, and $\times 2.0 \mathrm{~N} 4$ develop between 10 and 20 small anticyclonic vortices at the latitude spaced evenly around the longitude. The spacing of the emerging vortices is not directly related to

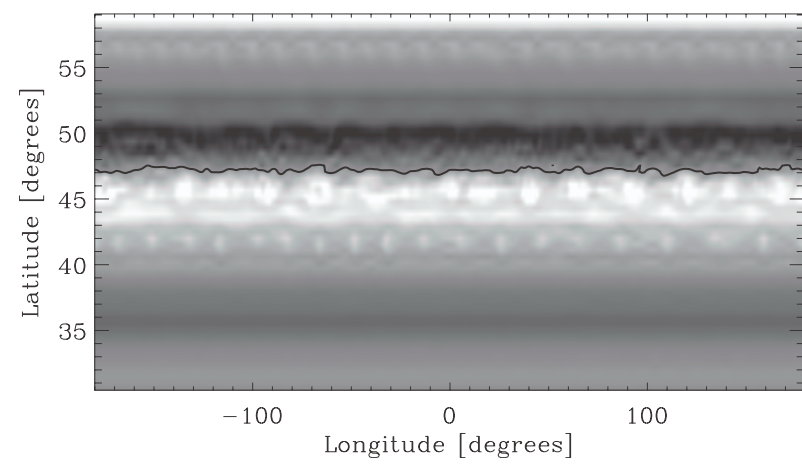

FIG. 16. The relative vorticity map of the case $\times 1.0 \mathrm{~N} 4$ at hour 2400 of the simulation on the model layer near the $100-\mathrm{hPa}$ level, showing the entire horizontal simulation domain. The relative vorticity increases from white to gray to black, and the solid line follows the jet's core. The SoPs-like chain of anticyclonic vortices appears at $41.5^{\circ} \mathrm{N}$ latitude as white blobs.

the meandering of the $47^{\circ} \mathrm{N}$ jet because the SoPs region is well outside of the domain affected by the Ribbon dynamics. In our simulations, the depth of these vortices seems to be limited by the simulation domain and to reach down to the 5-bar level.

Choi et al. (2009) note that the SoPs resides in a narrow range of latitude around $40^{\circ} \mathrm{N}$ and the average spacing between the pearls is about $4^{\circ}$ in longitude over a $100^{\circ}$ longitudinal section. They estimate that the uncertainties in the coordinates of the imaged features are $1^{\circ}-2^{\circ}$, so our SoPs-like feature, centered at $41.5^{\circ} \mathrm{N}$, is located within their error bars. Their infrared measurements are sensitive to features above $\sim 3$-bar level; thus, the vortices that form in our simulations are consistent with characteristics of the SoPs. However, the chain of anticyclones that emerge in our simulations is not a stable structure in the long term. Although the quasiperiodic structure lasts until the end of the 4800-h simulations, the anticyclonic vortices start to merge toward the end and result in a smaller number of larger vortices. If the SoPs are a long-lived feature, there must be a steady supply of anticyclones to maintain the structure for our model to be consistent; however, in such a scenario, it is unclear what process would keep the vortices to the observed small size without significantly growing through mergers.

\section{Comparison of the Ribbon to the Gulf Stream}

Meandering geophysical zonal jets have been studied extensively in the context of meandering ocean currents, especially the Gulf Stream (GS), which flows along the East Coast of the United States until it departs from the coast near Cape Hatteras. Figure 17 is a sea surface temperature map of the Gulf Stream region of the northern 


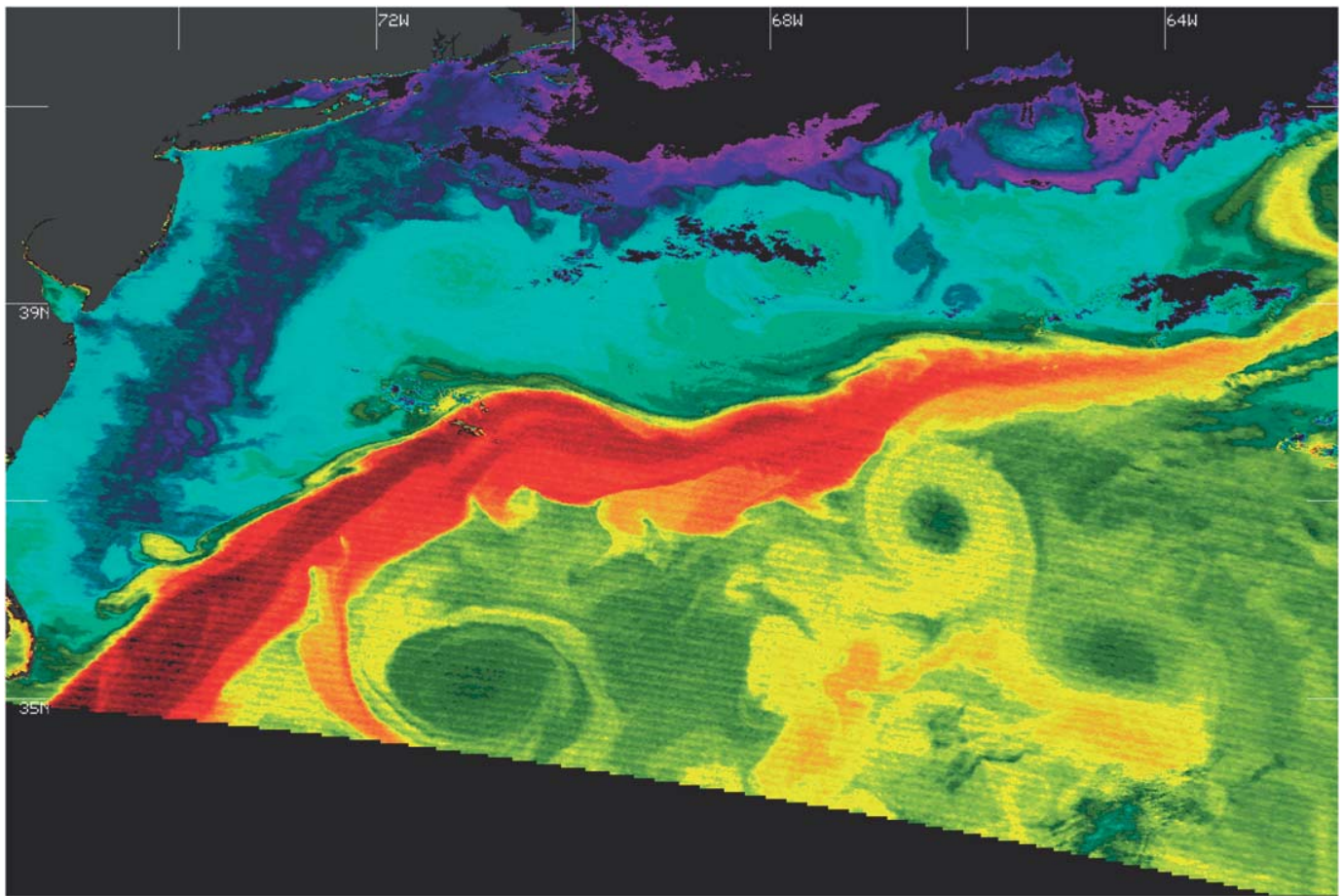

FIG. 17. The sea surface temperature map of the Gulf Stream region, obtained by the Moderate Resolution Imaging Spectroradiometer (MODIS) onboard the Earth Observing System Terra satellite. Red is warm and blue is cold. [Public domain image, National Aeronautics and Space Administration (NASA) Goddard Space Flight Center (GSFC).]

Atlantic, which shows that the warm water transported by the ocean current follows a sinuous path. It flows northeastward on average and plays a major role in the poleward oceanic heat transport in the northern Atlantic (Schmitz and McCartney 1993). In this section, we compare the observed and modeled characteristics of the meandering of the Gulf Stream and the Ribbon jet and argue that the two features share analogous geophysical fluid dynamic processes.

\section{a. Observed characteristics}

Both the Ribbon jet and the GS flow along a meandering path in which spatial oscillation is not dominated by a single oscillation mode and which contains a wide range of wavenumbers (for GS, Lee and Cornillon 1996; for the Ribbon, Sromovsky et al. 1983 and Godfrey and Moore 1986). The meandering propagates downstream in both GS and the Ribbon with respect to the rotating planet's frame of reference, and upstream with respect to the fluid parcels moving in the respective jet's core. The GS's path is known to shift its course continuously with time scales between about 1 and 10 days (Robinson et al. 1974). The propagation of the GS meandering does not keep the crests and the troughs coherent for more than several months (see Fig. 1 in Lee and Cornillon 1995). In comparison, although the Ribbon's time-dependent behavior has not been well resolved, Sromovsky et al. (1983) show a few crests and troughs losing coherence within the three Saturnian rotations covered in the report.

In GS, the meandering path often buckles and pinches off vortices, which can be either cyclonic or anticyclonic depending on the orientation of the buckling (Weatherly and Kelley 1985; Pratt and Stern 1986; Cornillon et al. 1994). No detachment of vortices has been reported in the Ribbon region; however, Godfrey and Moore (1986) report at least three major "kinks" in their dataset that mark buckling in the zonal jet like that shown in Fig. 2 . The GS meandering's crests and troughs often accompany a filamentary streak that protrudes from the point of maximum curvature in the path of GS as shown in Fig. 17. The images of the Ribbon region also show filamentary features as shown in Fig. 1.

The path of GS also accompanies a region of sharp horizontal temperature gradient (i.e., a thermal front), which was documented as early as in 1940 (Iselin 1940). The sharp relative vorticity gradient associated with GS coincides with the thermal front, and the result is a sharp PV gradient (Hall and Fofonoff 1993; Rossby and Zhang 2001; Logoutov et al. 2001; Meinen et al. 2009). The 
amplitude of the meandering in the cross-stream direction is much bigger than the width of the jet characterized by the width of this PV front. Read et al. (2009)'s PV analysis shows that the Ribbon is also a PV front. The amplitude of the Ribbon meandering, $\sim 2^{\circ}$ in latitude (Sromovsky et al. 1983), is comparable to the width of the PV front as resolved in Read et al.

\section{b. Modeling of meandering jets}

Geophysical fluid dynamic studies of GS meandering have focused on the narrowness of its PV front. For example, in an approach called the thin jet model, the equations of motion are simplified and linearized assuming that the width of the PV front is negligible to derive an equation that describes the jet's path (e.g., Robinson et al. 1975; Flierl and Robinson 1984; Pratt 1988; Cushman-Roisin et al. 1993). Another approximation called the contour dynamics model takes a piecewise constant PV field and studies the dynamics of the interface between regions of different PV (e.g., Meacham 1991). The formulations and results of thin jet and contour dynamic models are summarized by Flierl (1999). In particular, Meacham (1991) and Flierl (1999) showed that for the buckling and pinching of a zonal jet to occur, the baroclinic effect must be large enough that the instability's phases are unaligned in the vertical. However, as both thin jet and contour dynamic models do not allow homogenization of PV, they do not allow for a complete study of the development of the instability's nonlinear saturation. There have also been many linear and quasi-linear stability analyses relevant to meandering jets (e.g., Meacham and Flierl 1991; Boss et al. 1996; Paldor and Ghil 1997; Boss and Thompson 1999), which are useful for examining the physical mechanisms of meandering instabilities but nevertheless not applicable to nonlinear growth and saturation.

To the best of our knowledge, Flierl et al. (1987) was the first to demonstrate that the instabilities in an unstable jet can nonlinearly saturate. Using a quasigeostrophic barotropic model, they tested the stability of various initial flows that contain single-mode perturbations and examined the instability growth. Their results first show that the instability modes' initial growth rates agree with linear stability analyses. They then demonstrate that on longer time scales the instability can nonlinearly saturate and equilibrate its amplitude. When the instability saturates in a simulation, the eddy component of the kinetic energy stops growing and chaotically fluctuates around an equilibration level. They also show that once the instability contains multiple modes, the meandering behavior becomes dominated by modemode interactions, and their Fig. 19 in particular shows many morphological characteristics suggestive of chaotic meandering. Their results are extended to ageostrophic flows using a shallow-water model by Poulin and Flierl (2003).

The equilibration of eddy kinetic energy in nonlinearly saturated instabilities has also been shown by Allen et al. (1991) in the context of the coastal transition zone jet, and Polavarapu and Peltier (1993) in the context of Earth's midlatitude atmospheric jet stream; however, the jets in these studies do not chaotically meander because the mode-mode interactions between multiple instability modes seem to be suppressed by the short simulation domains, which extend approximately one instability wavelength in the zonal (east-west) direction. In Swanson and Pierrehumbert (1994)'s calculations, instabilities saturate but do not show chaotic meandering. Swanson and Pierrehumbert report that in the lower layer the reversal of PV gradient in the initial condition is eliminated during the course of their simulations through PV homogenization, which results in a PV profile that does not violate the Charney-Stern stability criterion; perhaps this prevented the jet from chaotically meandering in Swanson and Pierrehumbert's experiments.

The meandering amplitude's nonlinear saturation after its nonlinear growth was, to our knowledge, first studied in detail by Sutyrin et al. (2001). Using a fully three-dimensional primitive equation model, their experiments confirm Meacham (1991)'s contour dynamics result that baroclinic effects are crucial in the buckling and pinching of a GS-like zonal jet and the detachment of vortices. They proceed to compare cases with flat and sloping bottoms in both $f$-plane and $\beta$-plane geometries to show that the sloping bottom and the planetary $\beta$ effect both act in suppressing the meandering instability's growth and amplitude. Their numerical experiments also show that the instability amplitude is greater for the cases with larger PV change across the PV front; when the chaotic meandering state is reached, some of the cases continue to violate the Charney-Stern criterion even though the instability partially homogenizes PV. Sutyrin et al. (2001) essentially demonstrate that both topographic and planetary $\beta$ effects have similar effects on the suppression of the meandering instability, which is consistent with the prediction made by linear studies such as Poulin and Flierl (2005). Frolov et al. (2004) further extends Sutyrin et al. (2001)'s investigation on the effect of the topographic $\beta$ effect on the GS meandering and reach a consistent result. Frolov et al. identify four stages of meandering instability growth similar to the LG, NG, and NS phases we described above in our simulations (in addition, they include the initial transient phase).

The growth and equilibration of meandering instability we found in our Ribbon jet simulations share many 
dynamic characteristics with the studies of meandering ocean currents. First, we showed that as the instabilities evolve from the LG into NG phases, the eddy kinetic energy growth is dominated by the baroclinic component, and when the jet meanders chaotically in the NS phase, the instabilities develop substantial baroclinic activities as illustrated in Figs. 6 and 10, which is consistent with the findings by Meacham (1991) and Sutyrin et al. (2001). Once the Ribbon jet reaches the chaotic meandering state, the crests and troughs do not propagate coherently for more than several hundred hours, as illustrated in Fig. 7a. Also, the growth of $K_{\text {Eddy }}$ in our Ribbon jet simulations is analogous to the behaviors shown in the studies of jets in Earth's atmosphere and oceans; as discussed above, eddies grow in an unstable jet until a saturation point is reached, after which $K_{\text {Eddy }}$ chaotically fluctuates. Our results also agree with Sutyrin et al. (2001) that the instability amplitude depends on the magnitude of the PV change across the PV front associated with the zonal jet. We also demonstrated that when the PV change across Saturn's $47^{\circ} \mathrm{N}$ jet is set large enough, the jet can buckle, pinch, and shed cyclonic and anticyclonic eddies. Although buckling has been seen in the Ribbon's cloud patterns (Fig. 2), vortex detachment has not been observed on Saturn's Ribbon jet; however, we note that vortex detachments occurred in most of our simulations that produced a meandering jet resembling the observed Ribbon wave.

\section{Discussion}

Our study was motivated by the Voyager observations of Saturn's atmospheric zonal jet at $47^{\circ} \mathrm{N}$, which showed the jet to be in a meandering state (Smith et al. 1982). We tested Godfrey and Moore (1986)'s hypothesis that the wavy structure arises through baroclinic instabilities. We examined the stability of the $47^{\circ} \mathrm{N}$ jet through full 3D nonlinear simulations and confirmed that the observed jet profile is indeed susceptible to instabilities with a substantial baroclinic component. Our numerical experiments show that the instabilities in the jet grow until their amplitudes reach saturation, after which point the jet remains in a chaotically meandering state resembling the cloud morphology observed on Saturn along the $47^{\circ} \mathrm{N}$ jet. By the time a Ribbon-like meandering develops, the jet's vertical shear becomes similar to that derived by Read et al. (2009). Above the 100-hPa level, the eddies grow to substantial amplitudes in our simulations. The Cassini Composite Infrared Spectrometer (CIRS) has seen a similar large-amplitude quasi-periodic thermal structure at the latitude of the Ribbon (L. N. Fletcher 2009, personal communication). Fletcher estimates that the altitude of the quasiperiodic structure spans between 10 and $100 \mathrm{hPa}$ with a wavelength around $\sim 30^{\circ}$ in longitude, similar to results produced in our simulations in the stratosphere.

We interpret the saturation of the meandering amplitude to be a result of nonlinear effects in the interaction between the instability modes and the zonal mean state. The nonlinear effects can manifest in three possible ways. First, as the instability amplitude grows beyond the linear regime, the direct interaction between the instability modes and the background jet can halt the further instability growth. The chaotic meandering can still occur in this scenario through interaction between multiple instability modes. A second scenario is that as the instability amplitude becomes large, the instability modes break and act as a source of small-scale turbulence. The eddies in the turbulence can transport the energy either into or away from the jet. If the eddies act to pump the jet, that keeps the jet in an unstable state with active instabilities; the instabilities continue to act as a source of turbulence, which in turn cycles the energy back into the jet to maintain a closed steady-state process (in reality, dissipation, albeit weak, is a nonnegligible sink of energy). In the third scenario, if the instability-triggered turbulent eddies transport momentum away from the jet, the jet weakens until it reaches a linearly stable configuration in which steady freepropagating (most likely Rossby) waves remain.

Since the instabilities do not cause major changes in the jet's PV profile (as demonstrated in Fig. 11), we rule out the third scenario that the end-state jet is linearly stable. The PV homogenization triggered by the instabilities is minor at all altitudes as shown in Figs. $8 \mathrm{~b}$ and $11 \mathrm{~b}$ and the Charney-Stern stability condition is still violated when the system reaches the NS phase. Because the stability condition is a necessary but not sufficient condition, its violation does not necessarily guarantee instabilities, but the continued violation does illustrate that the system did not relax toward a linear stability limit when the instabilities saturate. However, we do note that the core of the $47^{\circ} \mathrm{N}$ jet relaxes toward neutral stability according to Arnol'd's second criterion as shown in Fig. 9. Based on these analyses, we rule out that the final state is linearly stable, but we are not able to conclude which of the first two scenarios is dominant in making the instabilities saturate in our simulations.

Over a longer time scale, on the real planet, forcing and dissipation likely play major roles in maintaining the meandering; our study's focus is to show that a Ribbonlike meandering is compatible with the observed state of the $47^{\circ} \mathrm{N}$ jet, and its maintenance is beyond our scope, although our simulations do demonstrate that the meandering can be sustained without forcing over hundreds of Saturnian rotation periods. 
We then showed that the growth rate and the amplitude of the meandering instability depend on the magnitude of the sharp rise in PV, or the PV front, across the eastward jet stream. When the PV front is nonexistent (i.e., the jet does not cause a reversal in the north-south PV gradient), the instability simply does not occur (e.g., case $\times 0.1 \mathrm{~N} 4)$. The $47^{\circ} \mathrm{N}$ jet develops the meandering instability when a sharp PV front causes reversals in the PV gradient. Our experiment illustrates that the instability growth has three distinct phases. In the first phase, which we called the linear growth, the instability is dominated by a single zonal wavenumber, the sinusoidal oscillation propagates coherently, and the eddy kinetic energy undergoes an exponentially rapid growth. The second phase, the nonlinear growth, starts when multiple instability modes start interacting and the spatial oscillation become out of phase in the vertical; during this phase, the jet's meandering path starts exhibiting chaotic morphology while the meandering amplitude continues to grow. The meandering amplitude stops growing when it reaches a saturation amplitude, which we call the nonlinear saturation phase, during which the creation of the eddies is balanced by their destruction through shearing by the jet and the planetary $\beta$ effect. In the NS phase, the meandering oscillation's phase does not vertically line up as in the NG phase. We also demonstrated that the growth and saturation of the instabilities are reflected in the temporal evolution of the eddy kinetic energy. Through variations in the background wind amplitude and the tropospheric static stability $N$, our simulations also show that the instability's growth rate and amplitude depend on the magnitude of the PV front.

In section 4, we illustrated the similarities between the Gulf Stream, a meandering ocean current on Earth, and Saturn's Ribbon jet through both observation and modeling. Both the GS and the Ribbon jet accompany a PV front (Logoutov et al. 2001; Read et al. 2009), and they both have many behavioral characteristics of a chaotically meandering jet. The extensive body of geophysical fluid dynamic studies on GS meandering provides a foundation for interpreting our Saturnian Ribbon modeling results. Theoretical and simple modeling studies show that chaotic meandering occurs when instability amplitudes saturate, which can happen in both a pure barotropic system (Flierl et al. 1987) and a system dominated by baroclinic effects (Sutyrin et al. 2001). In our simulations, the eddy baroclinic energy exceeds the eddy barotropic component, but nevertheless their magnitudes are similar when the instabilities saturate. The growth and saturation of the instabilities in our experiments follow stages similar to the temporal development of meandering modeled in the context of GS (Frolov et al. 2004). Based on these observed and modeled similarities between the GS and the Ribbon jet, we conclude that they are analogous phenomena resulting from instabilities in the PV fronts.

Our simulations also showed that, at $41.5^{\circ} \mathrm{N}$ latitude, the instabilities at the westward peak of the observed zonal wind profile produce a chain of anticyclonic vortices reminiscent of the String of Pearls discovered by Momary et al. (2006) using Cassini VIMS. The latitude corresponds to the region of maximum curvature in the zonal wind profile (i.e., maximum $\partial^{2} \bar{u} / \partial y^{2}$ ). The vorticities of the "pearls" have not been measured to date. Based on our simulations, we predict that each of the pearls is an anticyclonic vortex, similar to Jupiter's 5- $\mu \mathrm{m}$ hotspots, which are shown to be deep anticyclonic gyres by Showman and Dowling (2000). The chain of anticyclones that emerges in our simulations is in contrast to the general tendency that, at least on Jupiter, cloud clearings such as the pearls are cyclones while anticyclones are generally cloud-covered (Vasavada and Showman 2005). Also, the chain of anticyclones that forms at the latitude of the SoPs is not a stable configuration in our simulations as discussed in section $3 \mathrm{e}$, and the spacing between the pearls in our simulations (between $20^{\circ}$ and $40^{\circ}$ in longitude) is much greater than the observed $\sim 4^{\circ}$ longitudinal spacing (Choi et al. 2009).

Our numerical experiment supports one of the main conclusions of Read et al. (2009), namely that the large eddies in the atmospheres of the giant planets draw their energies from the zonal jets. This view is in contrast to studies of the zonal jet formation through inverse transfer of kinetic energy in the spectral space such as that of Sayanagi et al. (2008), who showed that robust zonal jets can naturally emerge, without any forcing, from small random eddies in the initial condition. In addition, observational measurements such as those made by Salyk et al. (2006; for Jupiter) and Del Genio et al. (2007; for Saturn, but not covering the Ribbon region) have also shown that the eddies pump momentum into the eastward jets; in other words, this picture shows that the zonal jets feed on the kinetic energy of the eddies. These two views are not necessarily in conflict. It is possible that the inverse energy transfer that feeds the zonal jets is in balance with the shedding of the kinetic energy by those jets through instabilities. When the kinetic energy is transferred from the mean flow to the eddies, the eddy kinetic energy can either undergo the inverse cascade and lead to larger eddy structures such as the stable large vortices on Jupiter, as demonstrated by Legarreta and Sánchez-Lavega (2008), or else eddy momentum transfer can pump the jet and cycle the energy back into the zonal flow. This view is similar to that expressed by Read et al. (2009); nevertheless, observational verification of this scenario requires a detailed 
analysis of the wind fields, which remains a challenge. A numerical model with realistic forcing and dissipation that consistently reproduces important features in the observed zonal jets and the large vortices will also be required to fully understand the atmospheric kinetic energy cycle on the giant planets.

We note that the temporal dynamics of the Ribbon jet have not been observed in detail. The propagation of the wavy structure has not been analyzed for longer than three Saturnian rotations, and whether the actual jet exhibits all behaviors we associate with chaotic meandering remains to be seen. No vortices have been observed to detach from the Ribbon jet, and the nature of the kinks in the jet's path reported by Godfrey and Moore (1986) remains unknown until their temporal behavior is resolved. To fully determine the three-dimensional PV structure of the jet will also require measurements of tropospheric static stability. These issues remain to be addressed by the ongoing Cassini and future missions to Saturn.

Acknowledgments. We thank the two anonymous reviewers for the extremely constructive comments. We also benefitted from extensive discussions with Dr. Timothy E. Dowling. This study has been supported by the Cassini Project.

\section{REFERENCES}

Allen, J. S., L. J. Walstad, and P. A. Newberger, 1991: Dynamics of the coastal transition zone jet. 2. Nonlinear finite amplitude behavior. J. Geophys. Res., 96, 14 995-15 016.

Arnol'd, V. I., 1966: On an a priori estimate in the theory of hydrodynamic stability (in Russian). Izv Vyssh. Uchebn. Zaved Matematika, 54, 3-5.

_ 1969: On an a priori estimate in the theory of hydrodynamic stability (in English). Amer. Math. Soc. Transl. Ser., 2, 267-269.

Boss, E., and L. Thompson, 1999: Mean flow evolution of a baroclinically unstable potential vorticity front. J. Phys. Oceanogr., 29, 273-287.

_, N. Paldor, and L. Thompson, 1996: Stability of a potential vorticity front: From quasi-geostrophy to shallow water. J. Fluid Mech., 315, 65-84.

Choi, D. S., A. P. Showman, and R. H. Brown, 2009: Cloud features and zonal wind measurements of Saturn's atmosphere as observed by Cassini/VIMS. J. Geophys. Res., 114, E04007, doi:10.1029/2008JE003254.

Cornillon, P., T. Lee, and G. Fall, 1994: On the probability that a Gulf Stream meander crest detaches to form a warm core ring. J. Phys. Oceanogr., 24, 159-171.

Cushman-Roisin, B., L. Pratt, and E. Ralph, 1993: A general theory for equivalent barotropic thin jets. J. Phys. Oceanogr., 23, 91-103.

Del Genio, A. D., J. M. Barbara, J. Ferrier, A. P. Ingersoll, R. A. West, A. R. Vasavada, J. Spitale, and C. C. Porco, 2007: Saturn eddy momentum fluxes and convection: First estimates from Cassini images. Icarus, 189, 479-492.
Dowling, T. E., 1995: Dynamics of Jovian atmospheres. Annu. Rev. Fluid Mech., 27, 293-334.

- A. S. Fischer, P. J. Gierasch, J. Harrington, R. P. Lebeau, and C. M. Santori, 1998: The Explicit Planetary IsentropicCoordinate (EPIC) atmospheric model. Icarus, 132, 221-238.

— , and Coauthors, 2006: The EPIC atmospheric model with an isentropic/terrain-following hybrid vertical coordinate. Icarus, 182, 259-273.

Flierl, G. R., 1999: Thin jet and contour dynamics models of Gulf Stream meandering. Dyn. Atmos. Oceans, 29, 189-215. , and A. R. Robinson, 1984: On the time-dependent meandering of a thin jet. J. Phys. Oceanogr., 14, 412-423.

— , P. Malanotte-Rizzoli, and N. J. Zabusky, 1987: Nonlinear waves and coherent vortex structures in barotropic $\beta$-plane jets. J. Phys. Oceanogr., 17, 1408-1438.

Frolov, S. A., G. G. Sutyrin, and I. Ginis, 2004: Asymmetry of an equilibrated Gulf Stream-type jet over topographic slope. J. Phys. Oceanogr., 34, 1087-1102.

Godfrey, D. A., and V. Moore, 1986: The Saturnian ribbon featureA baroclinically unstable model. Icarus, 68, 313-343.

Hall, M. M., and N. P. Fofonoff, 1993: Downstream development of the Gulf Stream from $68^{\circ}$ to $55^{\circ}$ W. J. Phys. Oceanogr., 23, 225-249.

Iselin, C. O'D., 1940: Preliminary report on long-period variations in the transport of the Gulf Stream system. Pap. Phys. Oceanogr. Meteor., 8, 1-40.

Lebeau, R. P., and T. E. Dowling, 1998: EPIC simulations of timedependent, three-dimensional vortices with application to Neptune's Great Dark Spot. Icarus, 132, 239-265.

Lee, T., and P. Cornillon, 1995: Temporal variation of meandering intensity and domain-wide lateral oscillations of the Gulf Stream. J. Geophys. Res., 100, 13 603-13 614.

$\longrightarrow$, and - 1996: Propagation of Gulf Stream meanders between $74^{\circ}$ and $70^{\circ}$ W. J. Phys. Oceanogr., 26, 205-224.

Legarreta, J., and A. Sánchez-Lavega, 2008: Vertical structure of Jupiter's troposphere from nonlinear simulations of long-lived vortices. Icarus, 196, 184-201.

Logoutov, O., G. Sutyrin, and D. R. Watts, 2001: Potential vorticity structure across the Gulf Stream: Observations and a PVgradient model. J. Phys. Oceanogr., 31, 637-644.

Lorenz, E. N., 1955: Available potential energy and the maintenance of the general circulation. Tellus, 7, 157-167.

Meacham, S. P., 1991: Meander evolution on piecewise-uniform, quasi-geostrophic jets. J. Phys. Oceanogr., 21, 1139-1170.

, and G. R. Flierl, 1991: Finite-amplitude waves on barotropic shear layers and jets. Geophys. Astrophys. Fluid Dyn., 56, 3-57.

Meinen, C., D. Luther, and M. Baringer, 2009: Structure, transport, and potential vorticity of the Gulf Stream at $68^{\circ} \mathrm{W}$ : Revisiting older data sets with new techniques. Deep-Sea Res. I, 56, 41-60.

Momary, T. W., and Coauthors, 2006: The zoology of Saturn: The bizarre features unveiled by the 5 micron eyes of Cassini/ VIMS. Bull. Amer. Astron. Soc., 38, 499.

Paldor, N., and M. Ghil, 1997: Linear instability of a zonal jet on an $f$ plane. J. Phys. Oceanogr., 27, 2361-2369.

Peixoto, J. P., and A. H. Oort, 1992: Physics of Climate. American Institute of Physics, $520 \mathrm{pp}$.

Polavarapu, S. M., and W. R. Peltier, 1993: The structure and nonlinear evolution of synoptic-scale cyclones. Part II: Wavemean flow interaction and asymptotic equilibration. J. Atmos. Sci., 50, 3164-3184.

Poulin, F. J., and G. R. Flierl, 2003: The nonlinear evolution of barotropically unstable jets. J. Phys. Oceanogr., 33, 2173-2192. 
, and -2005 : The influence of topography on the stability of jets. J. Phys. Oceanogr., 35, 811-825.

Pratt, L. J., 1988: Meandering and eddy detachment according to a simple (looking) path equation. J. Phys. Oceanogr., 18, 1627-1640.

— , and M. E. Stern, 1986: Dynamics of potential vorticity fronts and eddy detachment. J. Phys. Oceanogr., 16, 1101-1120.

Read, P. L., P. J. Gierasch, B. J. Conrath, A. Simon-Miller, T. Fouchet, and Y. H. Yamazaki, 2006: Mapping potentialvorticity dynamics on Jupiter. I: Zonal-mean circulation from Cassini and Voyager 1 data. Quart. J. Roy. Meteor. Soc., 132, 1577-1603.

— B. J. Conrath, L. N. Fletcher, P. J. Gierasch, A. A. SimonMiller, and L. Zuchowski, 2009: Mapping potential vorticity dynamics on Saturn: Zonal mean circulation from Cassini and Voyager data. Planet. Space Sci., 57, 1682-1698.

Robinson, A. R., J. R. Luyten, and F. C. Fuglister, 1974: Transient Gulf Stream meandering. Part I: An observational experiment. J. Phys. Oceanogr., 4, 237-255.

,$- \ldots$, and G. Flierl, 1975: On the theory of thin rotating jets: A quasi-geostrophic time dependent model. Geophys. Astrophys. Fluid Dyn., 6, 211-244.

Rossby, T., and H.-M. Zhang, 2001: The near-surface velocity and potential vorticity structure of the Gulf Stream. J. Mar. Res., 59, 949-975.

Salyk, C., A. P. Ingersoll, J. Lorre, A. Vasavada, and A. D. Del Genio, 2006: Interaction between eddies and mean flow in Jupiter's atmosphere: Analysis of Cassini imaging data. Icarus, 185, 430-442.

Sánchez-Lavega, A., 2002: Observations of Saturn's ribbon wave 14 years after its discovery. Icarus, 158, 272-275.

— J. F. Rojas, and P. V. Sada, 2000: Saturn's zonal winds at cloud level. Icarus, 147, 405-420.

Sayanagi, K. M., and A. P. Showman, 2007: Effects of a large convective storm on Saturn's equatorial jet. Icarus, 187, 520-539.
— - — , and T. E. Dowling, 2008: The emergence of multiple robust zonal jets from freely evolving, three-dimensional stratified geostrophic turbulence with applications to Jupiter. J. Atmos. Sci., 65, 3947-3962.

Schmitz, W. J., and M. S. McCartney, 1993: On the North Atlantic circulation. Rev. Geophys., 31, 29-49.

Showman, A. P., and T. E. Dowling, 2000: Nonlinear simulations of Jupiter's 5-micron hot spots. Science, 289, 1737-1740.

Skamarock, W. C., and J. B. Klemp, 1992: The stability of time-split numerical methods for the hydrostatic and the nonhydrostatic elastic equations. Mon. Wea. Rev., 120, 2109-2127.

Smith, B. A., and Coauthors, 1982: A new look at the Saturn system: The Voyager 2 images. Science, 215, 504-537.

Sromovsky, L. A., H. E. Revercomb, R. J. Krauss, and V. E. Suomi, 1983: Voyager 2 observations of Saturn's northern mid-latitude cloud features-Morphology, motions, and evolution. J. Geophys. Res., 88, 8650-8666.

Sutyrin, G. G., I. Ginis, and S. A. Frolov, 2001: Equilibration of baroclinic meanders and deep eddies in a Gulf Streamtype jet over a sloping bottom. J. Phys. Oceanogr., 31, 20492065.

Swanson, K., and R. T. Pierrehumbert, 1994: Nonlinear wave packet evolution on a baroclinically unstable jet. J. Atmos. Sci., 51, 384-396.

Tyler, G. L., V. R. Eshleman, J. D. Anderson, G. S. Levy, G. F. Lindal, G. E. Wood, and T. A. Croft, 1982: Radio science with Voyager 2 at Saturn: Atmosphere and ionosphere and the masses of Mimas, Tethys, and Iapetus. Science, 215, 553-558.

Vasavada, A. R., and A. P. Showman, 2005: Jovian atmospheric dynamics: An update after Galileo and Cassini. Rep. Prog. Phys., 68, 1935-1996.

Weatherly, G. L., and E. A. Kelley, 1985: Storms and flow reversals at the HEBBLE site. Mar. Geol., 66, 205-218. 